\title{
Retracted: Fluid Flow Simulations Based on an Equation-Solving Solution Gradient Strategy
}

\author{
Mathematical Problems in Engineering \\ Received 9 November 2014; Accepted 9 November 2014; Published 11 November 2014 \\ Copyright (C 2014 Mathematical Problems in Engineering. This is an open access article distributed under the Creative Commons \\ Attribution License, which permits unrestricted use, distribution, and reproduction in any medium, provided the original work is \\ properly cited.
}

The paper titled "Fluid Flow Simulations Based on an Equation-Solving Solution Gradient Strategy" [1] published in Mathematical Problems in Engineering has been retracted as it is found to contain a substantial amount of materials from the previously published papers for one of the coauthors: (1) Y. H. Hwang, "Development of a compact and accurate discretization for incompressible navier-stokes equations based on an equation-solving solution gradient, part I: kernel scheme fundamentals," Numerical Heat Transfer B, vol. 58, no. 3, pp. 145-169, 2010; (2) Y. H. Hwang, "Development of a compact and accurate discretization for incompressible navier-stokes equations based on an equation-solving solution gradient, part II: formulation on unstructured polygonal grids," Numerical Heat Transfer B, vol. 58, no. 3, pp. 170192, 2010; (3) Y. H. Hwang, "Development of a compact and accurate discretization for incompressible Navier-Stokes equations based on an equation-solving solution gradient, part III: fluid flow simulations on staggered polygonal grids," Numerical Heat Transfer B, vol. 58, no. 3, pp. 193-215, 2010.

\section{References}

[1] H.-S. Huang and Y.-H. Hwang, "Fluid flow simulations based on an equation-solving solution gradient strategy," Mathematical Problems in Engineering, vol. 2013, Article ID 409846, 19 pages, 2013. 


\title{
Fluid Flow Simulations Based on an Equation-Solving Solution
} Gradient Strategy

\author{
Ho-Shuenn Huang ${ }^{1}$ and Yao-Hsin Hwang ${ }^{2}$ \\ ${ }^{1}$ Department of Naval Architecture and Ocean Engineering, National Kaohsiung Marine University, Kaohsiung 811, Taiwan \\ ${ }^{2}$ Department of Marine Engineering, National Kaohsiung Marine University, Kaohsiung 811, Taiwan.
}

Correspondence should be addressed to Ho-Shuenn Huang; hoshuenn@webmail.nkmu.edu.tw

Received 22 February 2013; Accepted 12 April 2013

Academic Editor: Chang-Hua Lien

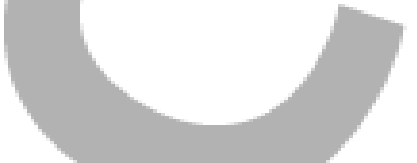

Copyright (C) 2013 H.-S. Huang and Y.-H. Hwang. This is an open access article distributed under the Creative Commons Attribution License, which permits unrestricted use, distribution, and reproduction in any medium, provided the original work is properly cited.

A compact and accurate discretization for fluid flow simulations is introduced in this paper. Contrary to the common wisdom in a convectional scheme, the solution gradient required for a high-resolution scheme is provided by solving its corresponding difference equation rather than by interpolation from solution values at neighboring computational nodes. To achieve this goal, a supplementary equation and its associated control volume are proposed to retain a compact and accurate discretization. Scheme essentials are exposed by numerical analyses on simple one-dimensional modeled problems to reveal its formal accuracy. Several test problems are solved to illustrate the feasibility of present formulation. From the obtained numerical results, it is evident that the proposed scheme will be a useful tool to simulate fluid flow problems in arbitrary domains.

\section{Introduction}

To provide a feasible simulation tool for incompressible flow in a complicated domain, we have successfully developed associated solution procedures on arbitrary LagrangianEulerian (ALE) $[1,2]$ and subsequently staggered triangular grid systems [3,4]. Nevertheless, its formal accuracy is quite limited and can be proved to be only first-order with the truncation error analyses [5]. Since a high-resolution scheme is demanded in practical flow simulations, it will be beneficial to raise its discretization accuracy and simulation efficiency.

In a finite volume method to simulate the convectiondiffusion equation, solution gradient in the computational cell must be appropriately defined to yield a high-order accurate representation of convection term $[6,7]$. Meanwhile, solution gradient must also be defined at the computational cell boundary to realize the diffusion term [8]. These solution gradients are generally obtained with the Taylorseries expansion based on the solution values at neighboring computational nodes, which will be an easy task on a regular grid. However, difficulties may be arisen if it is applied on an unstructured mesh to cope with a complicated domain. This process may bring on some computational disadvantages in the solution procedure.

(1) The resulting expression for solution gradient is indifferent to the peculiarity of governing equation.

(2) It will result in an extended difference stencil.

(3) Its accuracy heavily depends upon grid arrangement.

To circumvent these inconveniences, we will employ an alternative approach to determine the solution gradient: it should be directly calculated by solving its corresponding governing equation just as the solution variable. In this way, the characteristics pertaining to the governing equation can be directly deliberated in the solution gradient and consequently increase the simulation accuracy. To achieve this goal, the present paper is designed to unveil the underlying basics to be used in fluid flow problems. It can be summarized as follows:

(1) introducing a supplementary equation for solution gradient,

(2) constructing a control volume for the supplementary equation, 


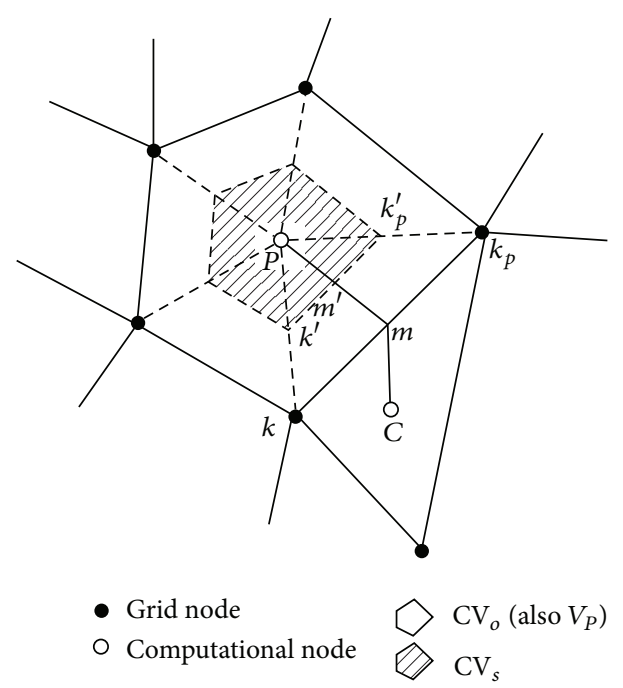

(a) Control volumes

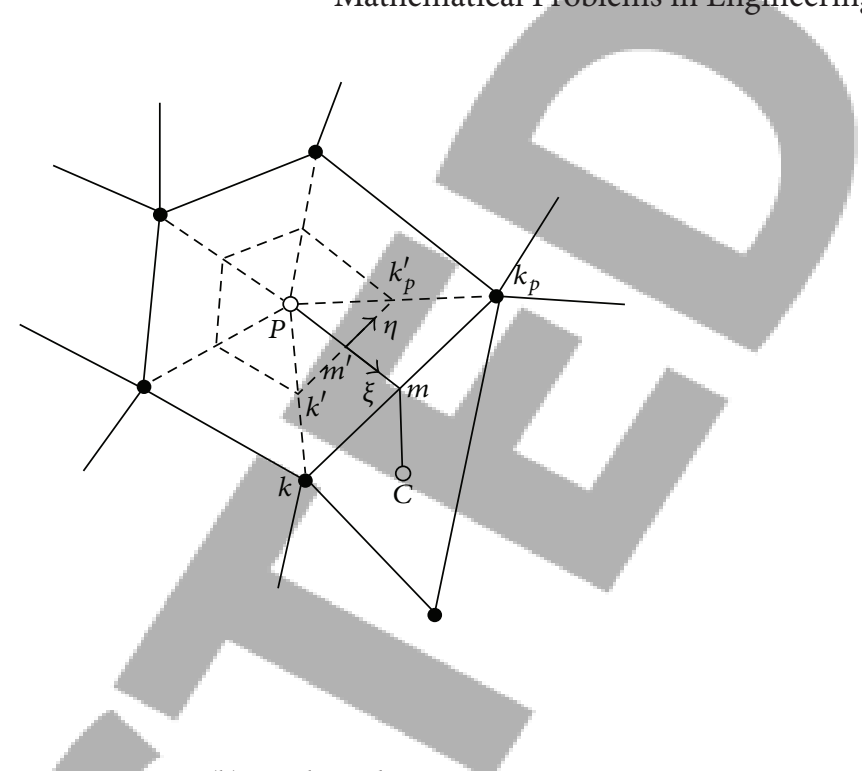

(b) Local coordinate

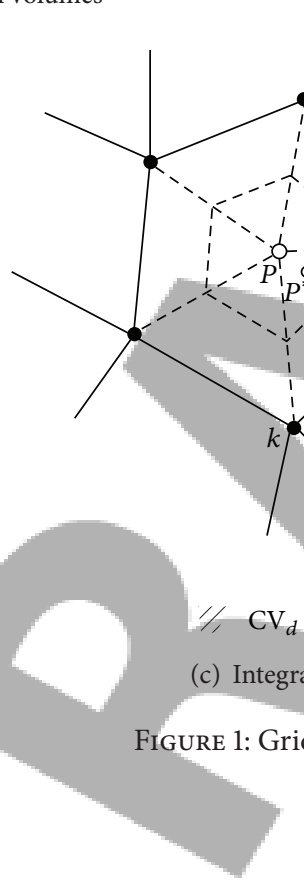

(3) establishing a practical expression for diffusion flux at cell boundaries,

(4) conducting numerical analyses on simple modeled problems,

(5) detailing the solution procedure in fluid flow calculations,

(6) verifying the solution accuracy by solving test problems,

It is noted that only the essential ingredients are delineated in the present paper. Detailed descriptions on associated formulation fundamentals, numerical analyses, solution procedure and more extensive validations can be consulted in our previous work [9-13].

\section{Formulation}

2.1. Governing Equations. Governing equations for incompressible flow can be generalized by the following convectiondiffusion-source form:

$$
\nabla \cdot \widehat{\mathbf{f}}=S,
$$

with flux function $\widehat{\mathbf{f}}=\rho \mathbf{v} \Phi-\mu \nabla \Phi$. To make the differential equation (1) numerically tractable, the interested domain is divided into nonoverlapping polygons in the finite volume formulation. Figure 1(a) shows a typical polygon delegated by a set of irregularly distributed grid nodes. The computational node for solution variable is assigned as the centroid of its associated polygon. Meanwhile, solution variable is mimicked by a combination of piecewise linear distributions:

$$
\Phi(\mathbf{r}) \approx \sum_{P=1}^{n_{C}} \phi(\mathbf{r} ; P)
$$


where $\phi(\mathbf{r} ; P)$ is the locally supported approximate linear function in association with $P$ th computational cell,

$$
\phi(\mathbf{r} ; P)= \begin{cases}\phi_{P}+\nabla \phi_{P} \cdot\left(\mathbf{r}-\mathbf{r}_{P}\right) & \text { if } \mathbf{r} \in V_{P} \\ 0 & \text { otherwise }\end{cases}
$$

with $\nabla \phi_{P}=p_{P} \mathbf{i}+q_{P} \mathbf{j}$. Physically, parameters $\phi_{P}, p_{P}$, and $q_{P}$ can be related to the solution variable and its spatial derivatives at the computational node.

In the present proposition, these spatial derivatives are directly computed based on their respective governing equations. An additional equation set derived from the original equation (1) is employed as the transporting equations for solution gradients:

$$
\nabla \cdot(\nabla \widehat{\mathbf{f}})=\nabla S
$$

These are the supplementary equations whose discretizations are also of vital importance in our formulation.

2.2. Control Volumes and Flux-Balanced Forms. In the finite volume formulation, difference equation is derived by integrating the governing equations over their corresponding control volumes. The control volume for original equation (1) is designated as the computational cell, $\mathrm{CV}_{o, P}=V_{P}$, which will ensure global conservation for solution variable. Nevertheless, the control volume for the supplementary equation (3), $C V_{s, P}$, is chosen as a subset of $V_{P}$. These control volumes are displayed in Figure 1(a), where corner nodes of $\mathrm{CV}_{s, P}$ are located at the midpoints of computational and grid nodes. Therefore, we denote $\mathrm{CV}_{s, P}$ as the supplementary control volume. With standard procedure in finite volume methodology, the difference counterpart for original equation (1) can be expressed by the following flux balanced form:

$$
\sum_{m=1}^{n_{V}} \mathbf{f}_{m} \cdot \mathbf{A}_{m}=\bar{s}_{P} V_{P}
$$

where $\mathbf{f}_{m} \cdot \mathbf{A}_{m}$ resembles the volume flux across the $m$ th boundary surface of $\mathrm{CV}_{o, P}$ and $\bar{s}_{P} V_{P}$ is the resulting integration of source term over the control volume. Similarly, the flux balanced form for supplementary equation (3) reads

$$
\sum_{m^{\prime}=1}^{n_{V}}(\nabla \mathbf{f})_{m^{\prime}} \cdot \mathbf{A}_{m^{\prime}}=\sum_{m^{\prime}=1}^{n_{V}} s_{m^{\prime}} \cdot \mathbf{A}_{m^{\prime}},
$$

where $m^{\prime}$ denotes the boundary face of the supplementary control volume, $\mathrm{CV}_{s, P}$.

To express the flux derivative term, $(\nabla \mathbf{f})_{m^{\prime}} \cdot \mathbf{A}_{m^{\prime}}$, in (5) with flux functions, we introduce a local coordinate $(\xi, \eta)$ defined by the unit base vectors:

$$
\mathbf{e}_{\xi}=\frac{\mathbf{r}_{m}-\mathbf{r}_{P}}{\left|\mathbf{r}_{m}-\mathbf{r}_{P}\right|}, \quad \mathbf{e}_{\eta}=\frac{\mathbf{r}_{k}-\mathbf{r}_{k_{p}}}{\left|\mathbf{r}_{k}-\mathbf{r}_{k_{p}}\right|} .
$$

These unit vectors are illustrated in Figure 1(b) for reference. By manipulating the coordinate transformation between the

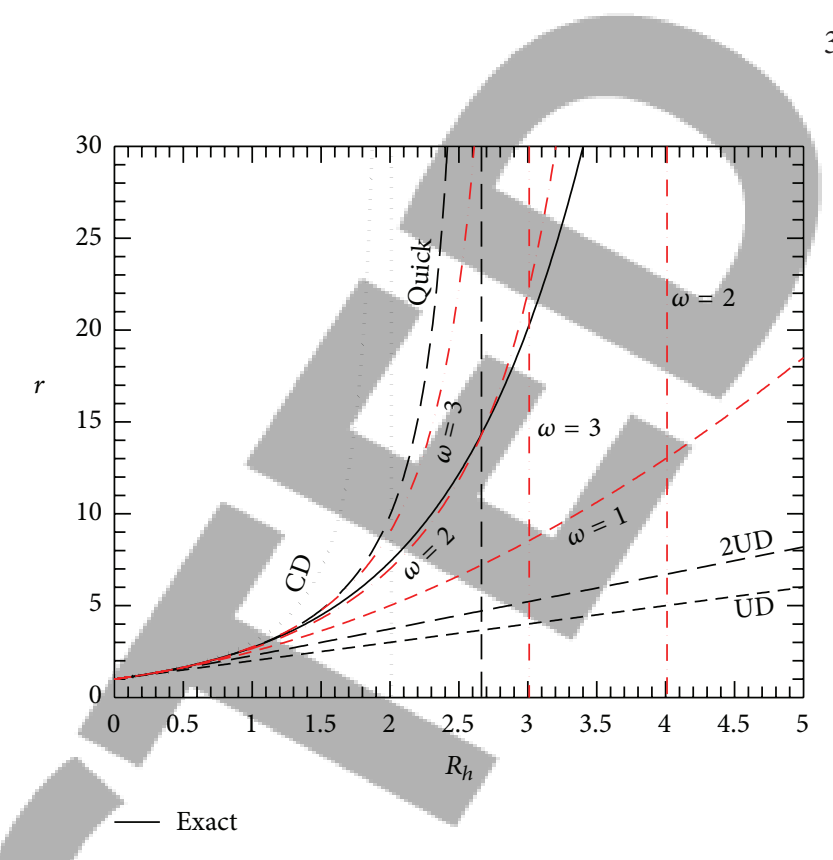

FIGURE 2: Increasing factors.

local and global systems, $(\nabla \mathbf{f})_{m^{\prime}}$ can be rearranged with the following expression:

$$
(\nabla \mathbf{f})_{m^{\prime}}=\frac{\mathbf{f}_{m}-\mathbf{f}_{P}}{\Delta \xi_{m P}} \nabla \xi+\frac{1}{2}\left(\nabla \mathbf{f}_{P}+\nabla \mathbf{f}_{m}\right) \cdot \frac{\partial \mathbf{r}}{\partial \eta} \nabla \eta .
$$

Substituting these terms into (5) will yield

$$
\begin{aligned}
\sum_{m=1}^{n_{V}} \frac{1}{\Delta \xi_{m p}} & {\left[\left(\mathbf{f}_{m} \cdot \mathbf{A}_{m}\right)-\left(\mathbf{f}_{P} \cdot \mathbf{A}_{m}\right)\right] \nabla \xi } \\
& +\frac{1}{2}\left\{\left[(\nabla \mathbf{f})_{P} \cdot \mathbf{A}_{m}+(\nabla \mathbf{f})_{m} \cdot \mathbf{A}_{m}\right] \cdot \frac{\partial \mathbf{r}}{\partial \eta}\right\} \nabla \eta \\
= & \sum_{m=1}^{n_{V}} s_{m^{\prime}} \mathbf{A}_{m} .
\end{aligned}
$$

In this way, (4) and (8) form a system of difference equations to imitate the original and supplementary equations (1) and (3), respectively.

2.3. Numerical Flux and Difference Equations. The numerical flux function at the computational node, $\mathbf{f}_{P} \cdot \mathbf{A}_{m}$, can be realized without difficulty, because the approximate solution (2a), (2b) is continuous at this point:

$$
\mathbf{f}_{P} \cdot \mathbf{A}_{m}=(\rho \mathbf{v})_{P} \cdot \mathbf{A}_{m} \phi_{P}-\mu_{P} \nabla \phi_{P} \cdot \mathbf{A}_{m} .
$$

However, at the computational cell boundary, it should be handled with care since the approximate solution (2a), (2b) becomes discontinuous. First, the upwind treatment is applied to derive the convection part:

$$
(\rho \mathbf{v} \phi \cdot \mathbf{A})_{m}=(\rho \mathbf{v} \cdot \mathbf{A})_{m}^{+} \phi\left(\mathbf{r}_{m} ; P\right)+(\rho \mathbf{v} \cdot \mathbf{A})_{m}^{-} \phi\left(\mathbf{r}_{m} ; C\right) .
$$




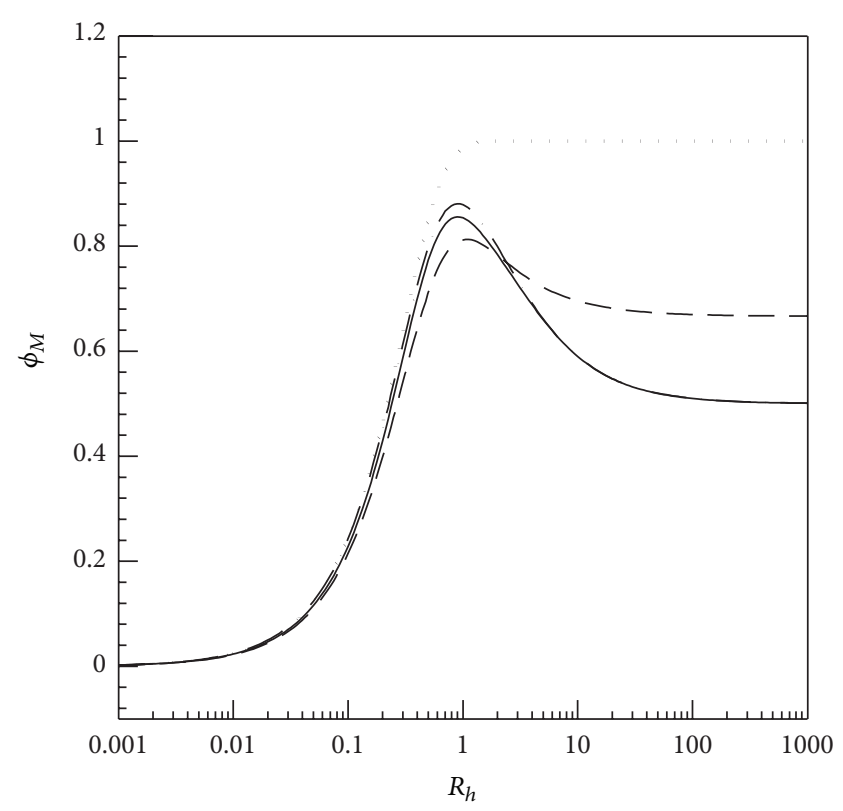

(a)

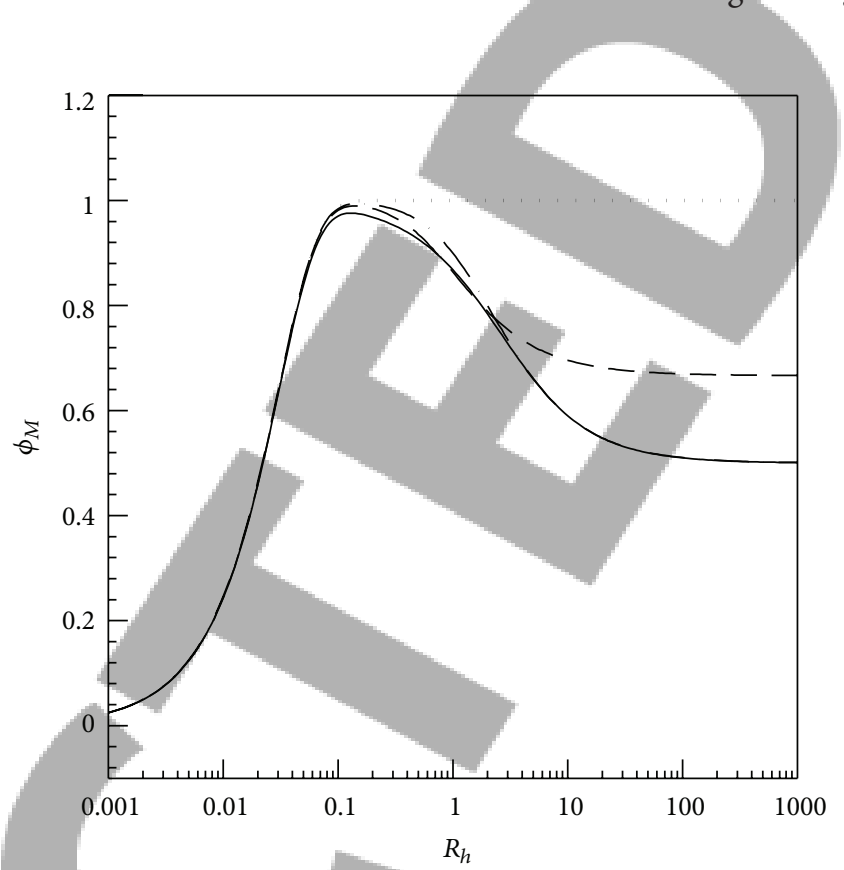

(b)

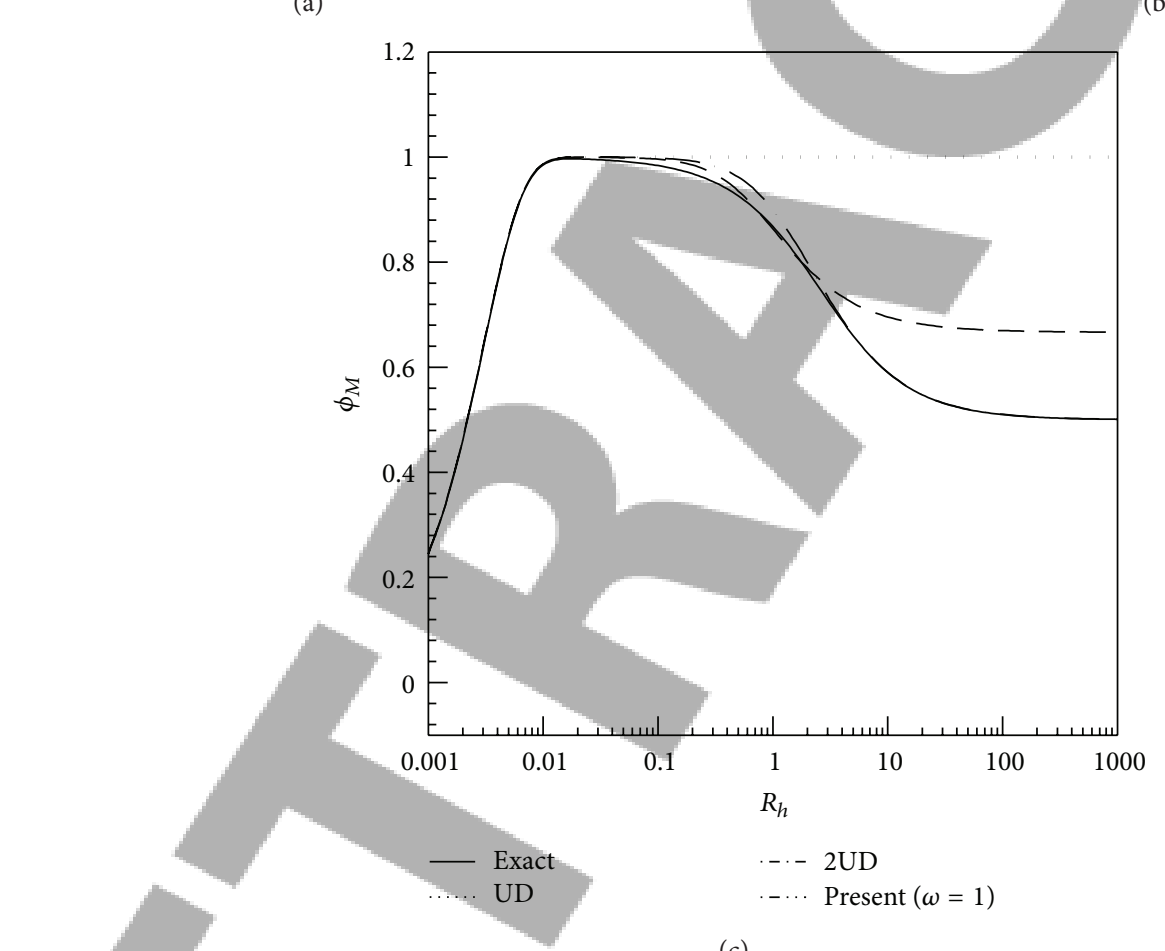

(c)

FIGURE 3: Solution of modeled problem.

Solution gradient in the diffusion term is resorted to the divergence theorem:

$$
\nabla \Phi_{m} \approx \nabla \phi_{m}=\frac{1}{\mathrm{CV}_{d}} \int_{\mathrm{CV}_{d}} \nabla \phi d V=\frac{1}{\mathrm{CV}_{d}} \int_{A\left(\mathrm{CV}_{d}\right)} \phi d \mathbf{A} .
$$

The integration volume, $\mathrm{CV}_{d}$, consists of a similar region as that enclosed by the lines connecting the grid and computational nodes surrounding the boundary surface, which is illustrated in Figure 1(c). This integration volume can be divided into two parts pertaining to neighboring computational cells:

$$
\mathrm{CV}_{d}=V_{P, m}+V_{C, m} .
$$

The resulting solution gradient reads

$$
\begin{aligned}
\nabla \phi_{m}= & \frac{V_{P, m}}{V_{P, m}+V_{C, m}} \nabla \phi_{P}+\frac{V_{C, m}}{V_{P, m}+V_{C, m}} \nabla \phi_{C} \\
& +\frac{\omega \mathbf{A}_{m}}{V_{P, m}+V_{C, m}}\left[\phi\left(\mathbf{r}_{m} ; C\right)-\phi\left(\mathbf{r}_{m} ; P\right)\right] .
\end{aligned}
$$




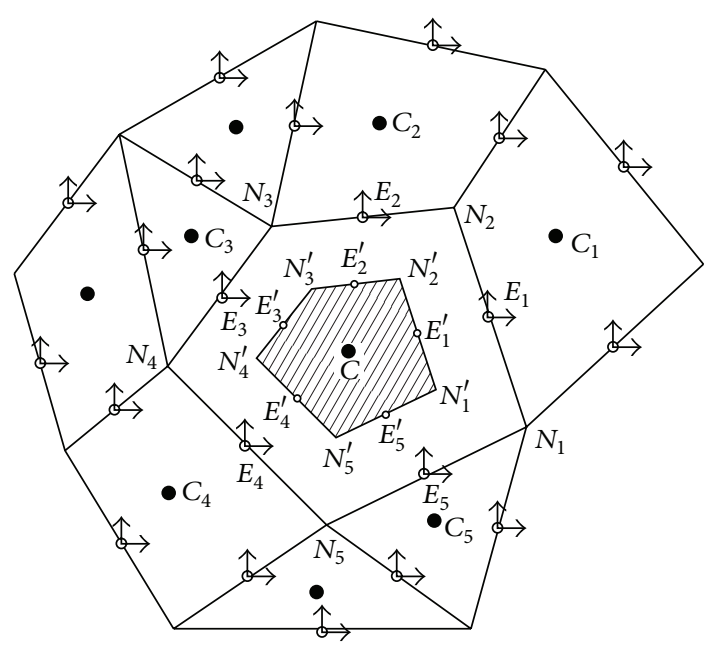

$\square \mathrm{CV}$ for continuity (scalar cell)

CV for supplementary continuity

$N$ : grid node

- C: scalar point (pressure node)

$\hat{\theta} \rightarrow$ : vector point (velocity node)

(a) Control volumes for scalar variable

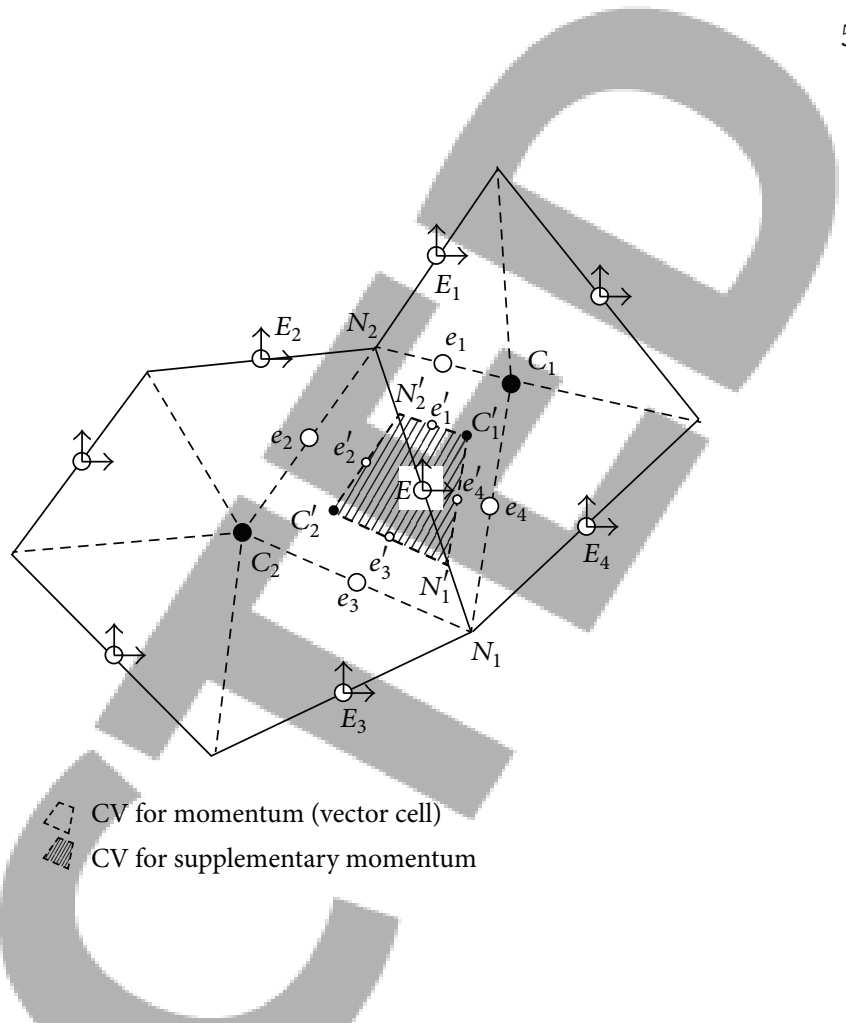

(b) Control volumes for vector variable

FIGURE 4: Staggered polygonal grid.

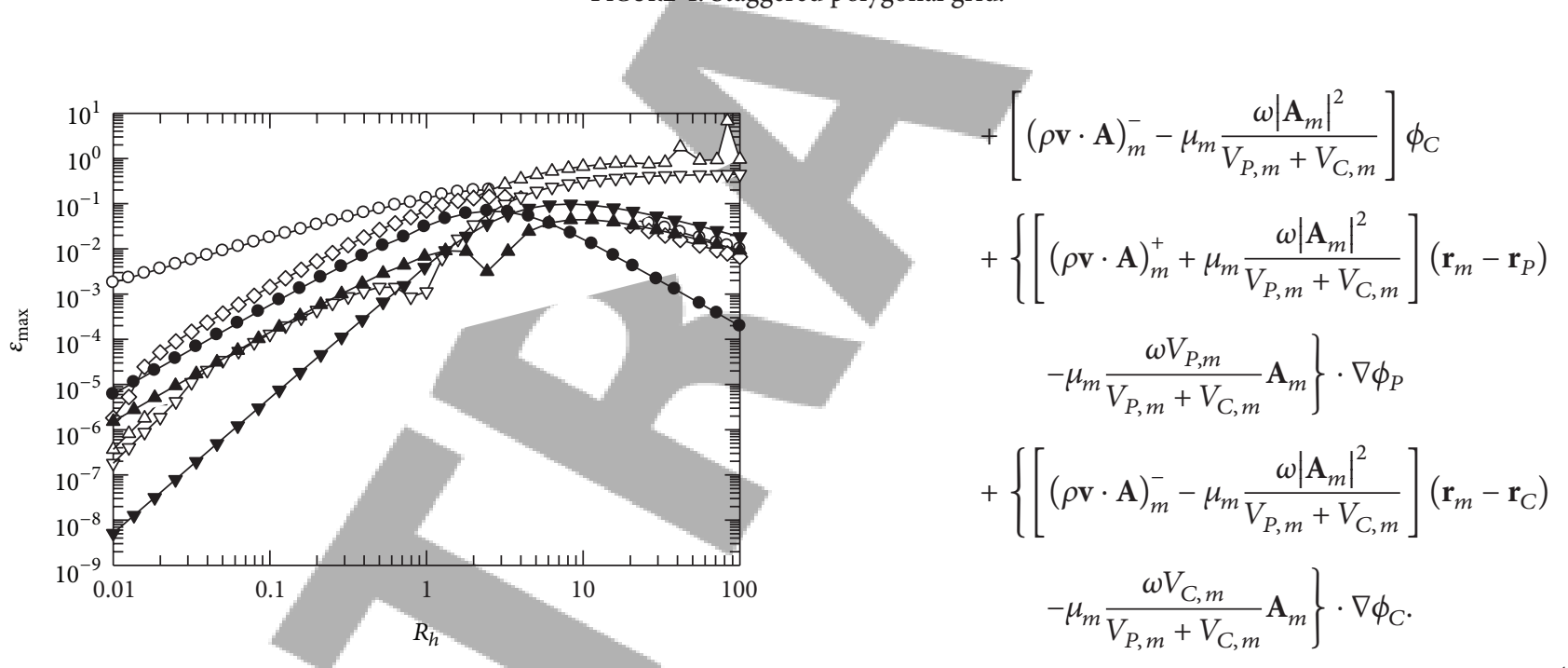

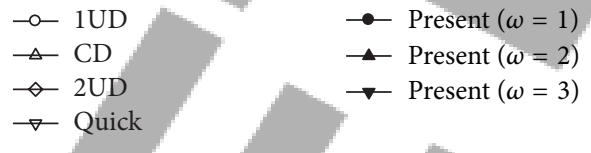

FIGURE 5: Solution error in linear convection-diffusion problem.

In the previous equation, scheme parameter $\omega$ indicates the integration factor to yield $\mathrm{CV}_{d}$. Further interpolations of this scheme factor will be given in a subsequent section.

Collecting the discretized convection and diffusion terms will lead to the resulting numerical flux term at cell boundary:

$$
\begin{aligned}
& \mathbf{f}_{m} \cdot \mathbf{A}_{m} \\
& \quad=\left[(\rho \mathbf{v} \cdot \mathbf{A})_{m}^{+}+\mu_{m} \frac{\omega\left|\mathbf{A}_{m}\right|^{2}}{V_{P, m}+V_{C, m}}\right] \phi_{P}
\end{aligned}
$$



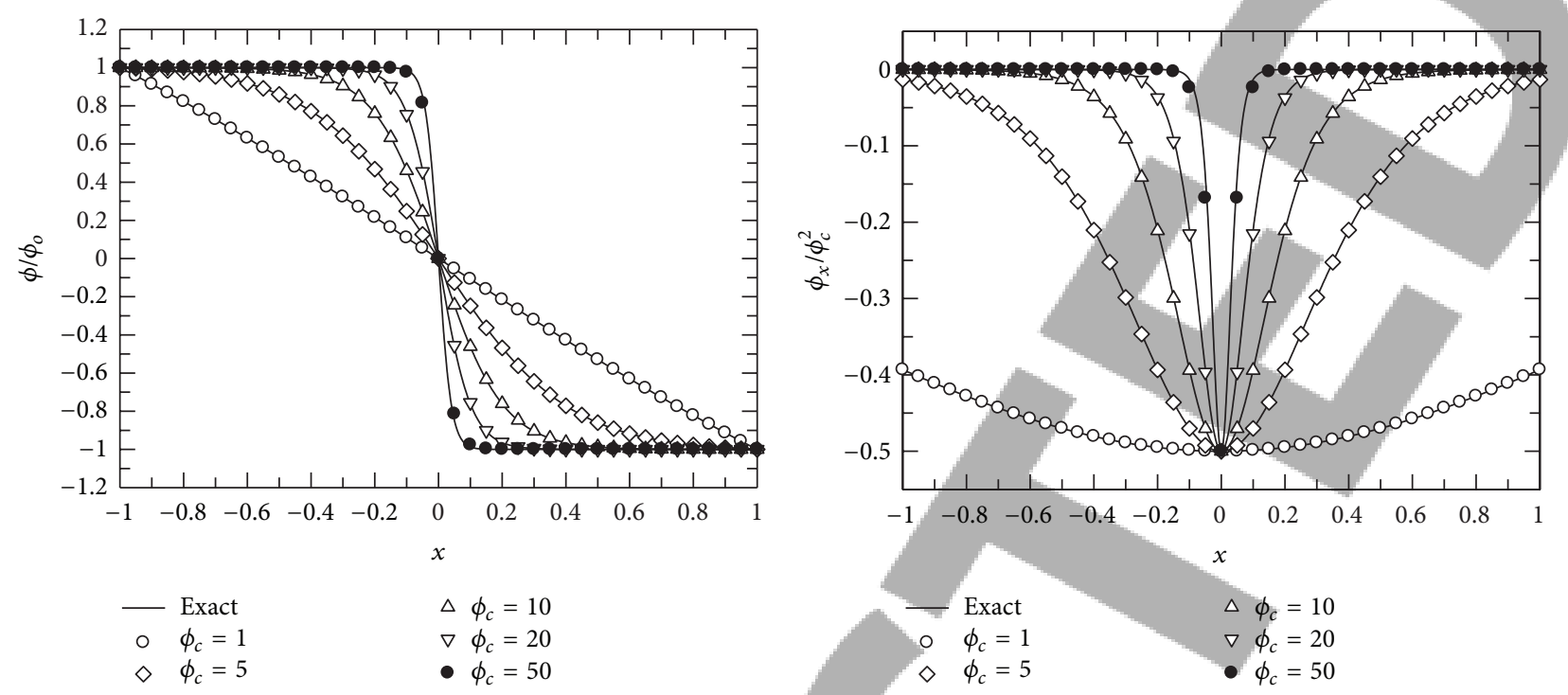

(a) Solution

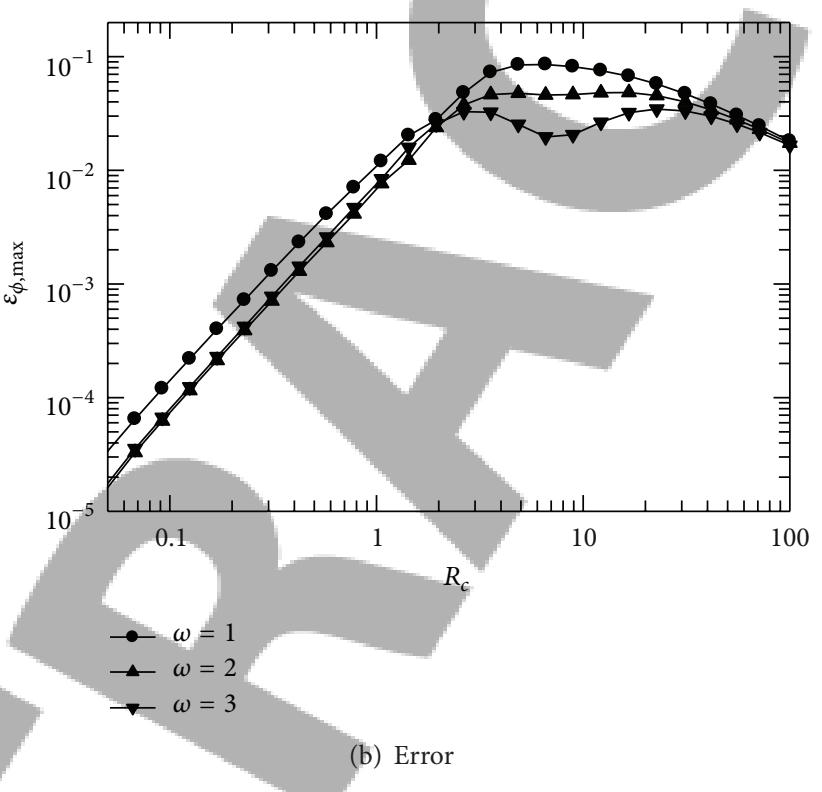

FIGURE 6: Solution and error distributions in one-dimensional second test problem.

By substituting these numerical functions into flux balanced equations (4) and (8), one can obtain the resulting difference equations for the original and supplementary governing equations, which can be expressed with the following form:

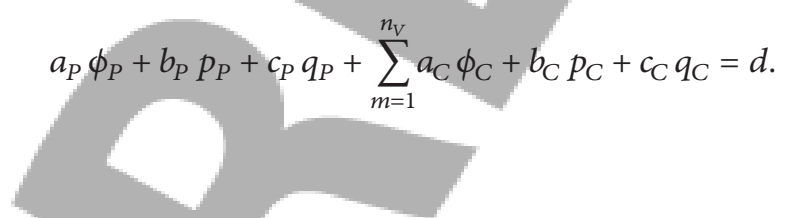

\section{Numerical Analysis}

From the previous derivations, the essential characteristics in our proposition can be summarized as follows:
(1) adoption of a supplementary equation to derive difference equation for solution gradient,

(2) selection of a particular control volume for the supplementary equation,

(3) usage of a variable integration range to obtain solution gradient at cell boundary.

With these special features, numerical analyses should be conducted to ensure its feasibility before it can be applied in more complicated circumstances.

3.1. Interpretation of Solution Gradient at Cell Boundary. In the one-dimensional linear problem, solution gradient at cell boundary (10b) can be simplified as 


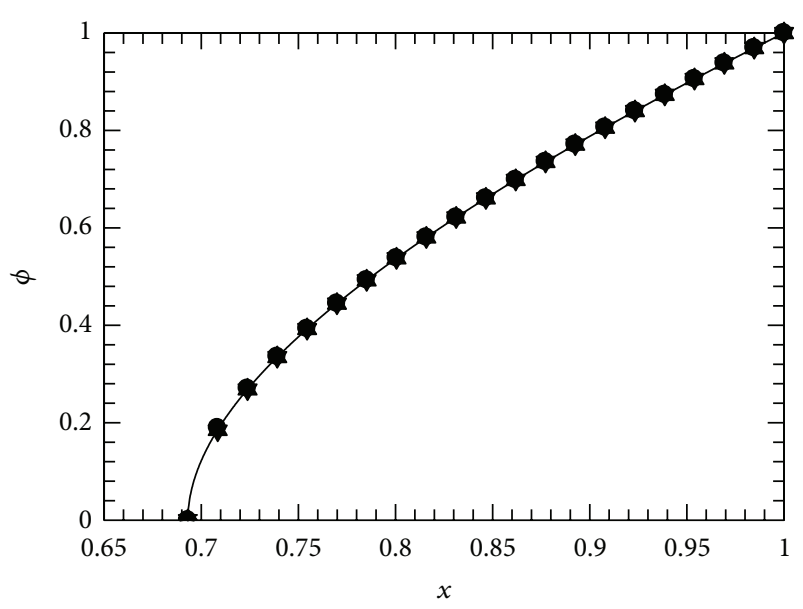

(a)

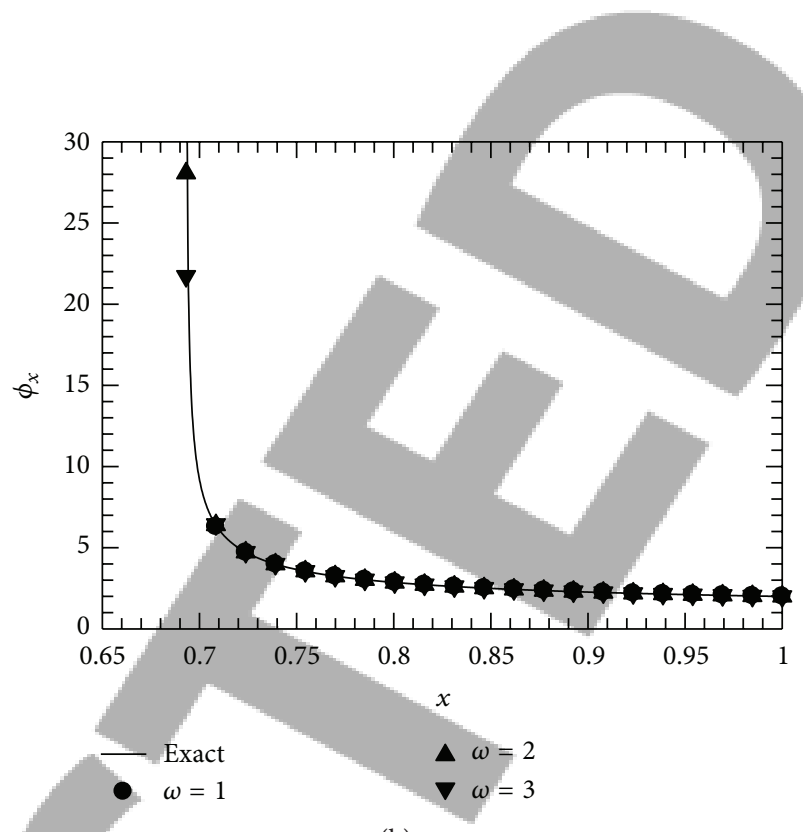

(b)

FIGURE 7: Solution distribution in one-dimensional third test problem.

$$
\begin{aligned}
\phi_{x, i+1 / 2}= & \frac{h_{i}}{h_{i}+h_{i+1}} p_{i}+\frac{h_{i+1}}{h_{i}+h_{i+1}} p_{i+1} \\
& +\frac{2 \omega}{h_{i}+h_{i+1}}\left[\phi\left(x_{i+1 / 2} ; i+1\right)-\phi\left(x_{i+1 / 2} ; i\right)\right] .
\end{aligned}
$$

(16a)

It can be interpreted as the volume averaged between two adjacent cells added by a penalty term arising from solution discontinuity. Therefore, besides the integration factor to yield integration volume, the scheme parameter $\omega$ can be regarded as a penalty factor in this expression. Meanwhile, it can also be rewritten as

$$
\begin{aligned}
\phi_{x, i+1 / 2}= & (1-\omega)\left(\frac{h_{i}}{h_{i}+h_{i+1}} p_{i}+\frac{h_{i+1}}{h_{i}+h_{i+1}} p_{i+1}\right) \\
& +\omega \frac{\left(\phi_{i+1}-\phi_{i}\right)}{h_{i} / 2+h_{i+1} / 2} .
\end{aligned}
$$

(16b)

This is a weighting average between those interpolated from solution variable and its gradient at computational nodes and $\omega$ stands for the weighting factor. Furthermore, this solution gradient can be further rearranged as:

$$
\phi_{x, i+1 / 2}=\frac{\phi\left(x_{i+1 / 2}+h_{i+1} / 2 \omega ; i+1\right)-\phi\left(x_{i+1 / 2}-h_{i} / 2 \omega ; i\right)}{h_{i} / 2 \omega+h_{i+1} / 2 \omega} \text { (16c) }
$$

This is a simple two-point representation and $\omega$ can then be designated as distance factor.

3.2. Increasing Factor. Consider the case with constant positive convective velocity and viscosity in a uniform grid system $\left(h_{i}=h\right)$. Difference equations can then be reduced as

$$
\mathbf{B} \Theta_{i-1}+\mathbf{A} \Theta_{i}+\mathbf{C} \Theta_{i+1}=\mathbf{d}_{i}
$$

with the solution vector, $\Theta=(\phi, p h / 2)^{T}$, and coefficient matrixes,

where $R_{h}=\rho u h / \mu$ is the cell Reynolds number. In the case of $\omega=1$, the solution gradient can be explicitly derived as

$$
p_{i, \omega=1}=\frac{2}{R_{h}+2}\left[\frac{\phi_{i+1}-\phi_{i}}{h}+\frac{h}{4 \mu}\left(s_{i+1 / 4}+2 \bar{s}_{i}-s_{i-1 / 4}\right)\right] .
$$

This expression clearly depicts that the solution gradient will adapt itself to flow condition (cell Reynolds number and source term). The resulting characteristic (nontrivial) increasing factor of our proposed scheme can be derived as [14]

$$
r=\frac{R_{h}^{2}+R_{h}+\omega\left(2+R_{h}\right)}{R_{h}+\omega\left(2-R_{h}\right)} .
$$

Obviously, the increasing factor will be dependent on the scheme factor, $\omega$. Meanwhile, the critical cell Reynolds 


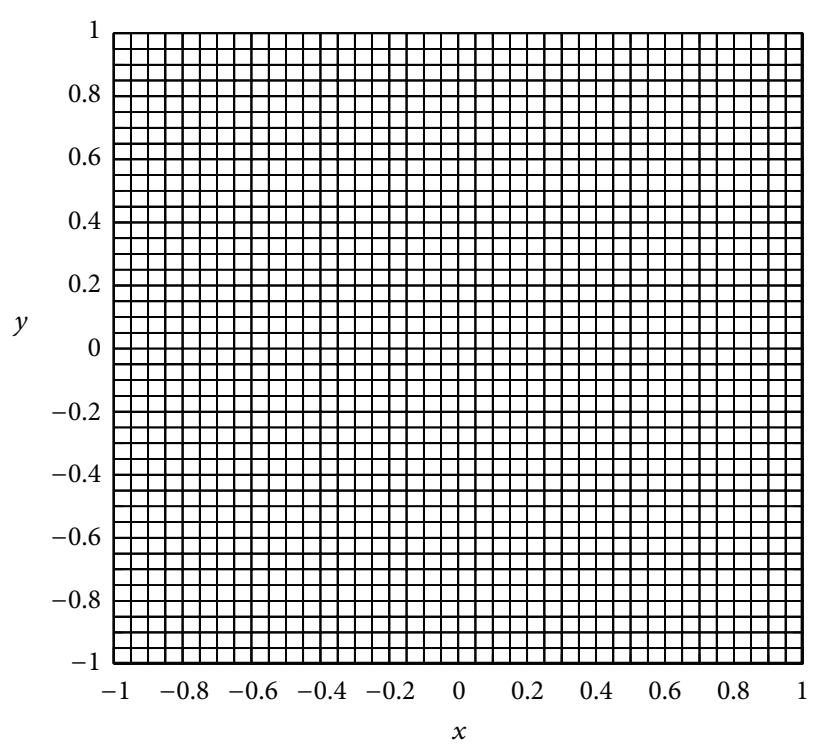

(a) Mesh A

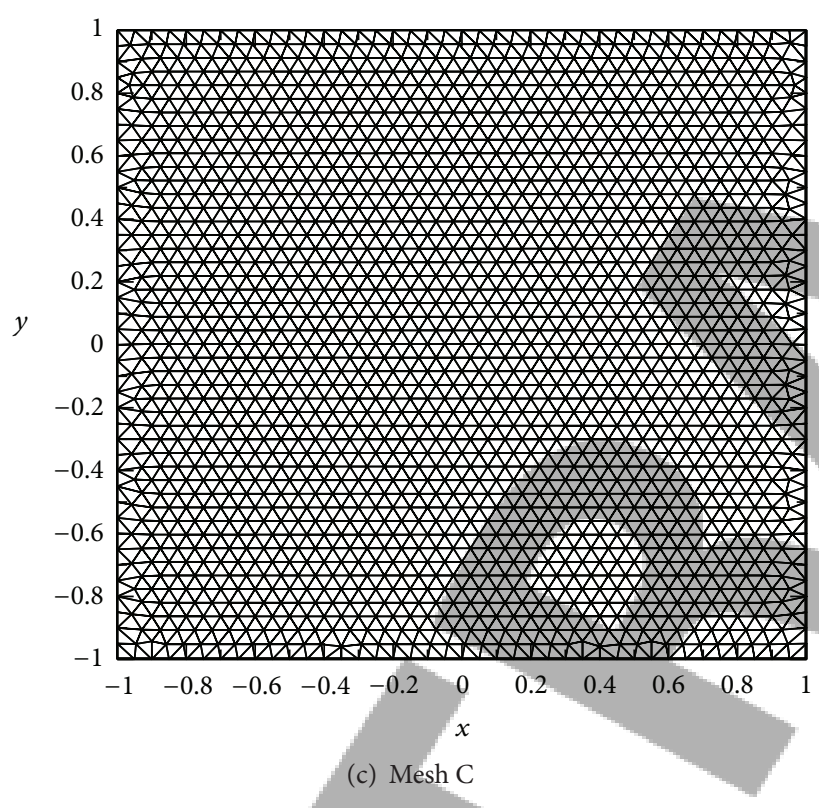

FIGURE 8: Four types of grid arrangement.

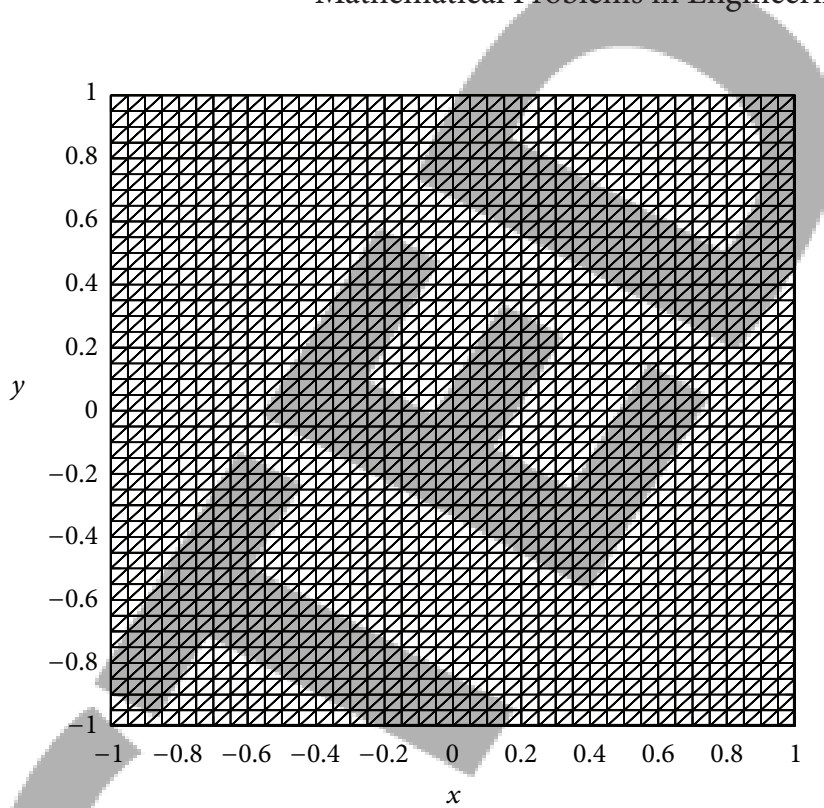

(b) Mesh B

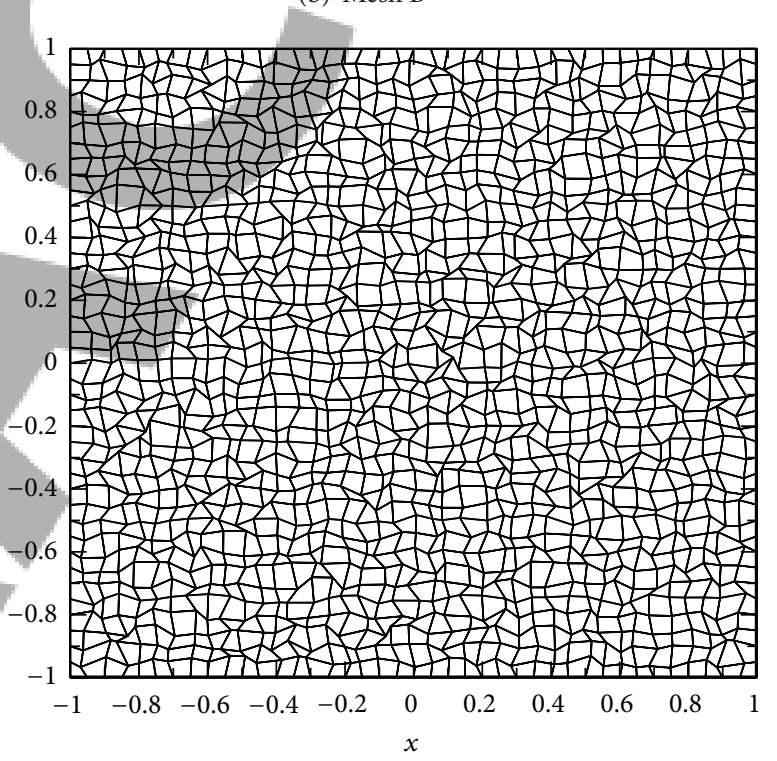

(d) Mesh D

To determine the possible range of the scheme factor, we turn to the exact increasing factor, $r_{\mathrm{ex}}=e^{R_{h}}$, which determines the exact scheme factor

$$
\omega_{\text {ex }}\left(R_{h}\right)=\frac{e^{R_{h}} R_{h}-R_{h}^{2}-R_{h}}{e^{R_{h}}\left(R_{h}-2\right)+R_{h}+2} .
$$

This is a monotone distribution with $\omega_{\mathrm{ex}}(0)=3$ and $\omega_{\mathrm{ex}}(\infty)=1$. Therefore, distribution range of this exact scheme factor is

$$
1 \leq \omega_{\mathrm{ex}} \leq 3 .
$$

This constraint is selected as the bound for scheme factor in our study. Figure 2 illustrates the distribution of increasing 


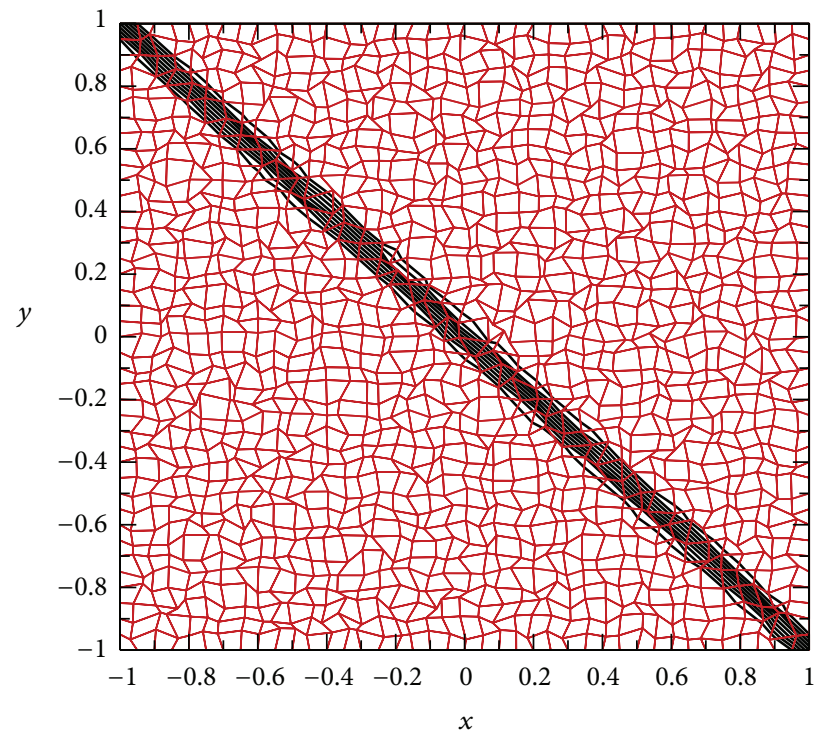

(a) Mesh D

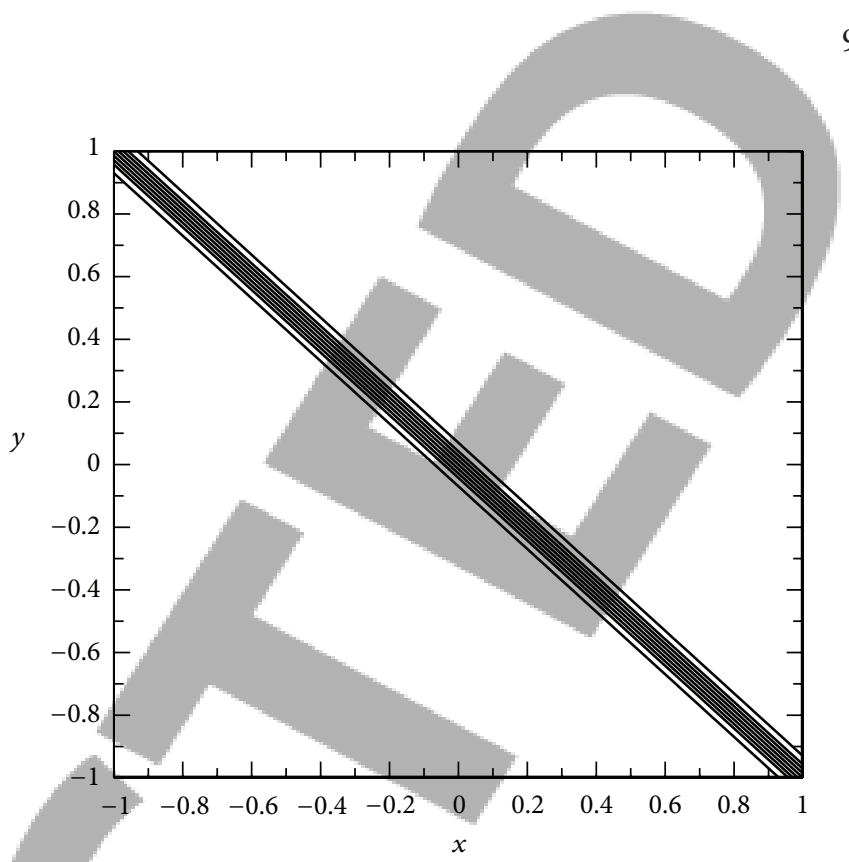

(b) Exact

FIGURE 9: Solution contours in two-dimensional first test problem.

factors for $\omega=1,2$ and 3 as well as other conventional schemes. It is clearly shown that the present formulation can provide more accurate increasing factor than the conventional schemes. In fact, the increasing factor (20a) can be approximated as

$$
\begin{aligned}
r= & 1+R_{h}+\frac{1}{2} R_{h}^{2}+\frac{1}{4}\left(1-\frac{1}{\omega}\right) R_{h}^{3} \\
& +\frac{1}{8}\left(1+\frac{1}{\omega^{2}}-\frac{2}{\omega}\right) R_{h}^{4}+\cdots,
\end{aligned}
$$

which is second-order accurate representation as compared with the exact one, $r_{\mathrm{ex}}$. Meanwhile, third-order accurate approximation can be obtained with $\omega=3$.

3.3. Solution of a Modeled Problem. Consider the modeled problem with the following source term:

with boundary conditions:

$$
S(x)= \begin{cases}\frac{\rho u}{h} & x_{M}-\frac{h}{2} \leq x \leq x_{M}+\frac{h}{2} \\ 0 & \text { otherwise, }\end{cases}
$$

$$
\Phi(0)=\Phi(1)=0 .
$$

That is, the source term exists within the computational cell located at $x=x_{M}$ and its strength increases as the grid spacing is refined. The total amount of source terms remain unchanged. This equation will demonstrate effects of existing source on solution characteristics. Exact and numerical solutions of this problem can be easily secured with simple arithmetic manipulations. Figure 3 depicts the

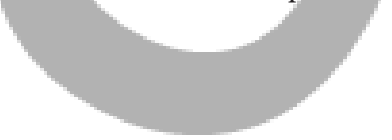

resulting solution at the source cell $\phi_{M}$ with respect to cell Reynolds number with various grid sizes. Generally speaking, the present formulation can provide more accurate results than UD and 2UD, especially when $R_{h}$ is large. In fact, as $R_{h} \rightarrow \infty$, it is easy to prove that UD and 2UD will approach to 1 and $2 / 3$, respectively, while both the exact solution and present formulation will approach to $1 / 2$.

\section{Fluid Flow Calculations}

4.1. Governing Equations. For convenience, the steady incompressible Navier-Stokes equations are expressed by the following conservation law form:

$$
\begin{gathered}
\nabla \cdot \widehat{\mathbf{f}}_{c}=0, \\
\nabla \cdot \widehat{\mathbf{f}}_{u}=-\frac{\partial p}{\partial x}, \\
\nabla \cdot \widehat{\mathbf{f}}_{v}=-\frac{\partial p}{\partial y},
\end{gathered}
$$

where mass, $x$ - and $y$-momentum flux functions read

$$
\begin{gathered}
\widehat{\mathbf{f}}_{c}=\rho \mathbf{v}, \\
\widehat{\mathbf{f}}_{u}=\rho \mathbf{v} u-\mu \nabla u, \\
\widehat{\mathbf{f}}_{v}=\rho \mathbf{v} v-\mu \nabla v .
\end{gathered}
$$



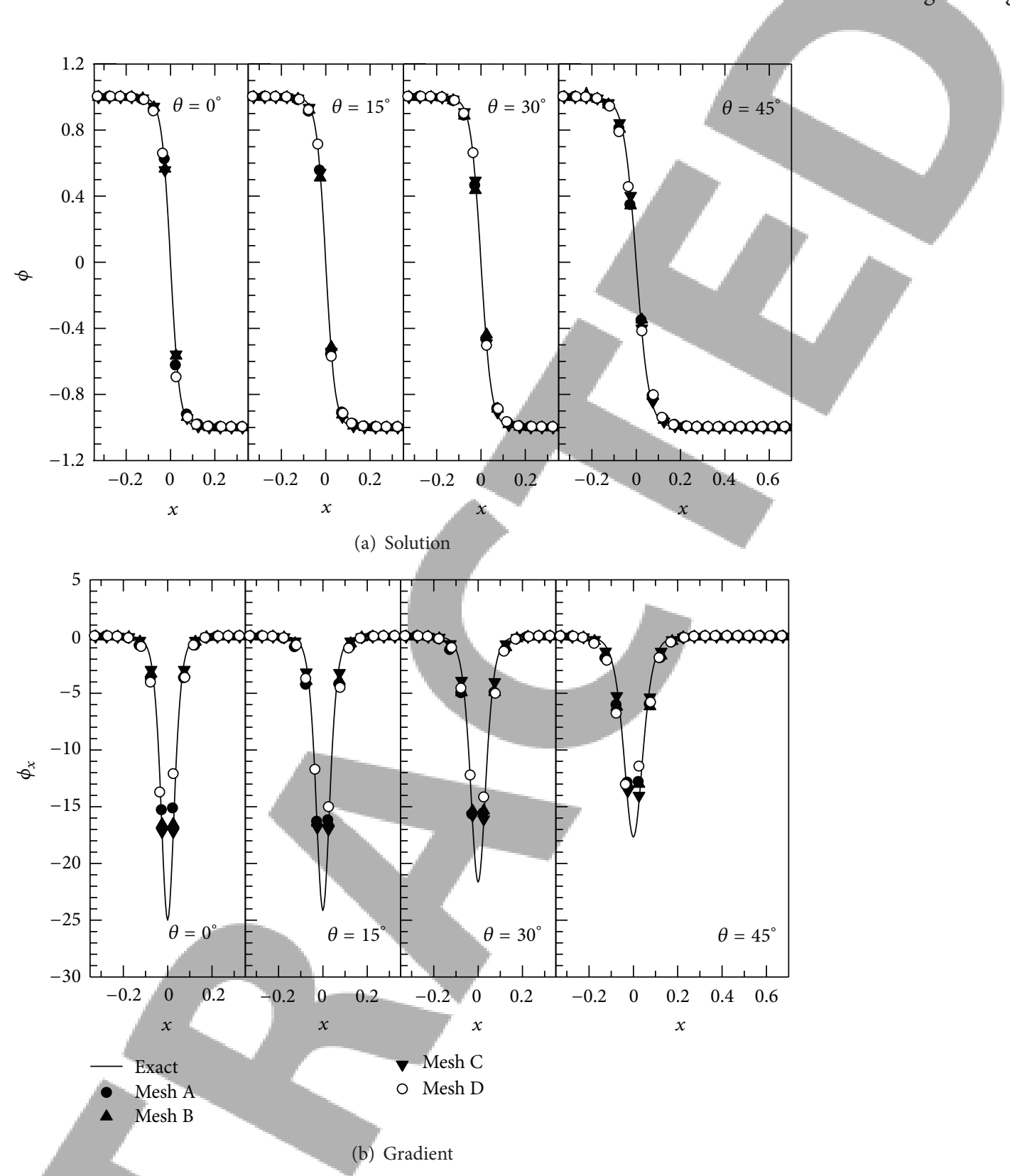

FIGURE 10: Solution profile along $y=0$ in two-dimensional first test problem.

The supplementary equations for solution gradients are derived from the original ones:

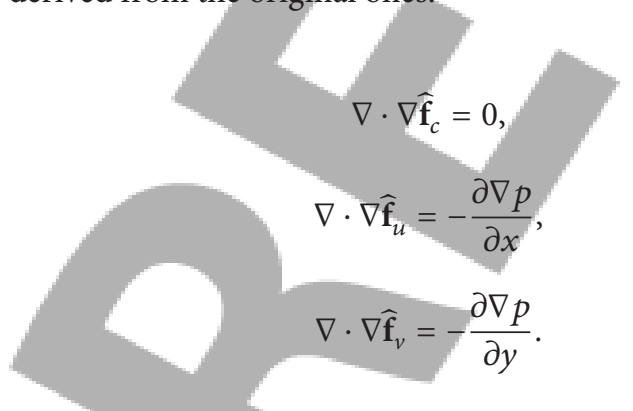

To derive the difference counterparts for these equations, the finite volume method is also employed by integrating the supplementary equations over their control volumes.
However, apart from those for original equations, the control volumes for these equations are specified in a different way.

4.2. Domain Discretization and Approximate Solutions. The interested domain is divided into nonoverlapping polygons in the finite volume formulation. Figure 4(a) shows some typical polygons delegated by a set of irregular grid nodes. These polygons are regarded as scalar cells $\left(V_{C}\right)$ and their centroids the scalar points. In our formulation, these centroids are assigned to be the computational locations for pressure, which are also designated as pressure nodes. Therefore, the control volume for continuity equation is coincident with the scalar cell. To prevent the occurrence of checkerboard pressure filed, we adopt a staggered polygonal grid system as shown in Figure 4(b). The computational locations for 


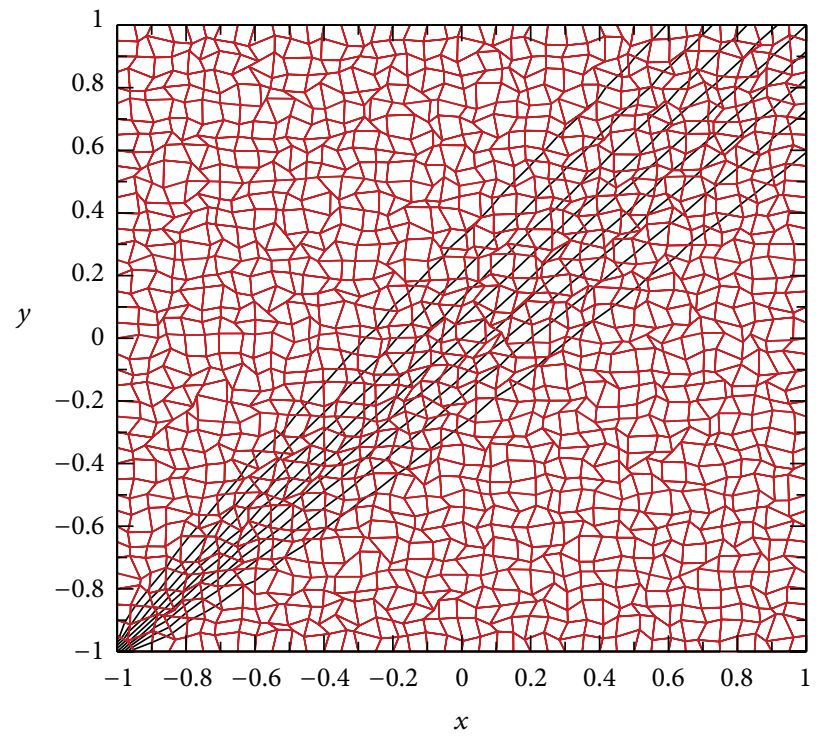

(a) Mesh D

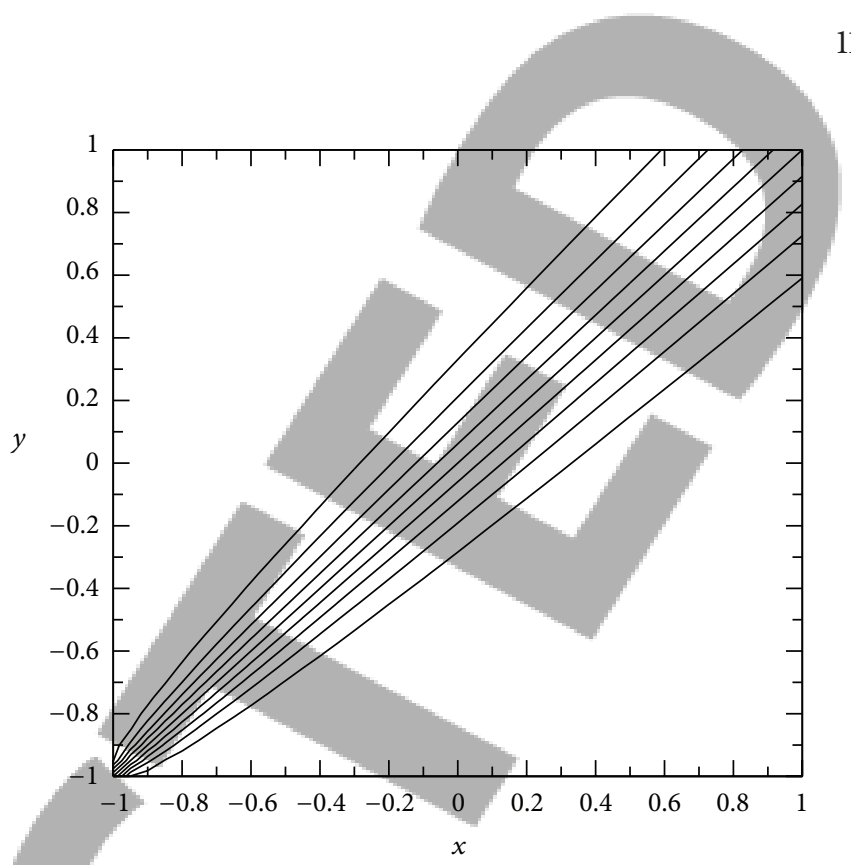

(b) Exact

FIGURE 11: Solution contours in two-dimensional second test problem.

velocity vector are staggered to those for pressure (pressure nodes). They are defined at the boundary face centers of scalar cells. For brevity, these locations are then designated as vector points or velocity nodes which are also demonstrated in Figures 4(a) and 4(b). The control volume associated with a velocity node is defined by connecting neighboring grid and pressure nodes. Figure 4(b) depicts a typical control volume for a velocity node, which is the region enclosed by four dashed lines connecting $C_{1}-N_{2}-C_{2}-N_{1}-C_{1}$ surrounding the velocity node, $E$. This control volume is regarded as the vector cell $\left(V_{E}\right)$ for momentum equation. From this particular arrangement, there are four boundary faces and two neighboring pressure nodes for a vector cell irrespective of the initial grid distribution.

To have a second-order accurate discretization, the approximate solutions for pressure and velocity vector are mimicked by a combination of piecewise linear distributions:

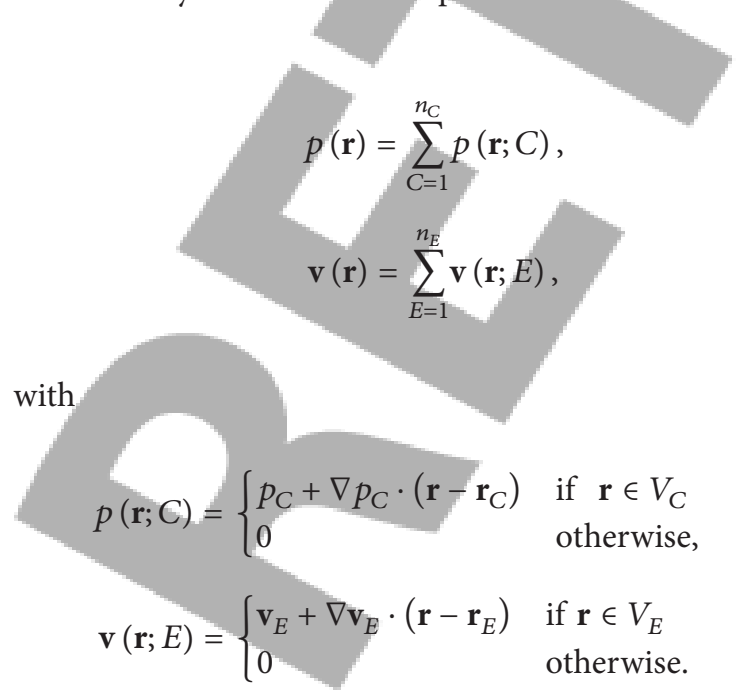

Following the derivation procedure given for a convectiondiffusion-source equation, the resulting difference equations for fluid flow calculations read

$$
\begin{gathered}
\sum_{E(C)}\left(\rho A_{x} \mathbf{U}+\rho A_{y} \mathbf{V}\right)_{E(C)}=\mathbf{0} \\
\mathbf{C}_{E} \mathbf{U}_{E}+\sum_{E e(E)} \mathbf{C}_{E e(E)} \mathbf{U}_{E e(E)}=\sum_{C(E)} \mathbf{D}_{C(E)}^{U} \mathbf{P}_{C(E)}, \\
\mathbf{C}_{E} \mathbf{V}_{E}+\sum_{E e(E)} \mathbf{C}_{E e(E)} \mathbf{V}_{E e(E)}=\sum_{C(E)} \mathbf{D}_{C(E)}^{V} \mathbf{P}_{C(E)},
\end{gathered}
$$

with unknown variable vectors:

$$
\begin{gathered}
\mathbf{P}=\left(p, p_{x}, p_{y}\right)^{T}, \quad \mathbf{U}=\left(u, u_{x}, u_{y}\right)^{T}, \\
\mathbf{V}=\left(v, v_{x}, v_{y}\right)^{T} .
\end{gathered}
$$

In the previous difference equations, $A_{x}$ and $A_{y}$ are the $x$ and $y$-direction components of surface vector $\mathbf{A}=A_{x} \mathbf{i}+A_{y} \mathbf{j}$, respectively. In addition, $C(E)$ and $E e(E)$ are the collection set of neighboring scalar and vector nodes for vector node, $E$, respectively, while $E(C)$ is the set of neighboring vector nodes for scalar node, $C$. As a reference shown in Figure 4(a), $E(C)=\left\{E_{1}, E_{2}, E_{3}, E_{4}, E_{5}\right\}$ and in Figure 4(b), $C(E)=\left\{C_{1}, C_{2}\right\}$ and $E e(E)=\left\{E_{1}, E_{2}, E_{3}, E_{4}\right\}$.

4.3. Pressure-Velocity Coupling and Solution Procedure. In the incompressible flow calculations, a pressure-velocity coupling relation must be established to proceed with the solution procedure [15]. In this study, we employ the SIMPLE algorithm to circumvent this difficulty. However, the conventional SIMPLE algorithm is used for pressure-velocity 

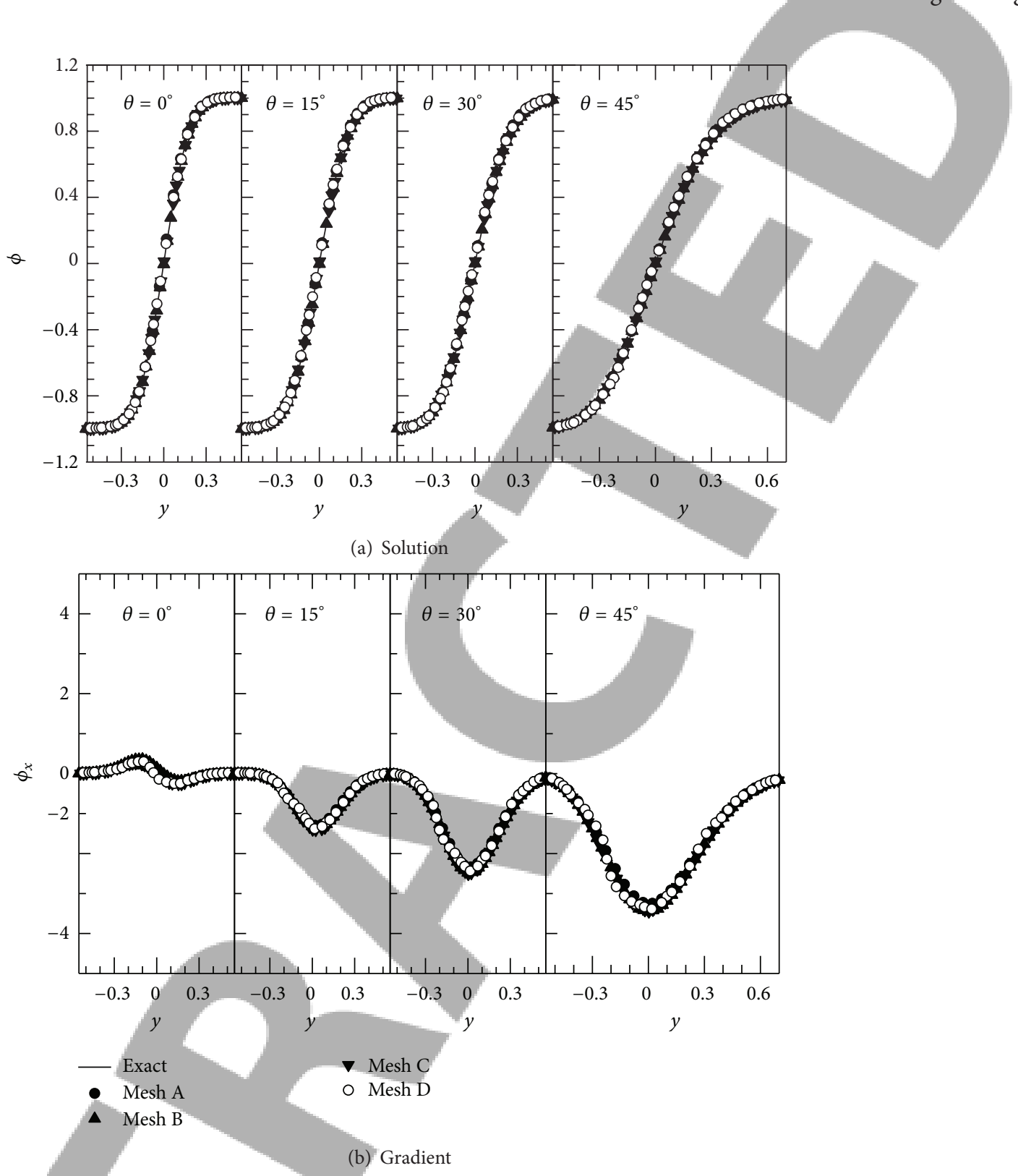

FIGURE 12: Solution profile along $x=0$ in two-dimensional second test problem.

coupling; it must be extended to include their derivatives as well in our formulation. First, all the solution variables and their derivatives are expressed with a tentative quantity added by a correction term:

$$
\mathbf{U}_{E}=\widetilde{\mathbf{U}}_{E}+\mathbf{U}_{E}^{\prime}, \quad \mathbf{V}_{E}=\widetilde{\mathbf{V}}_{E}+\mathbf{V}_{E}^{\prime}, \quad \mathbf{P}_{C}=\widetilde{\mathbf{P}}_{C}+\mathbf{P}_{C}^{\prime}
$$

In general, this tentative field $(\widetilde{\mathbf{U}}, \widetilde{\mathbf{V}}, \widetilde{\mathbf{P}})$ does not satisfy the continuity constraint and needed to be corrected by the correction term $\left(\mathbf{U}^{\prime}, \mathbf{V}^{\prime}, \mathbf{P}^{\prime}\right)$. The pressure-velocity coupling is built by the difference momentum equations (29b) and (29c) via their correction terms:

$$
\mathbf{U}_{E}^{\prime}=\mathbf{C}_{E}^{-1} \sum_{C(E)} \mathbf{D}_{C(E)}^{U} \mathbf{p}_{C(E)}^{\prime}, \quad \mathbf{V}_{E}^{\prime}=\mathbf{C}_{E}^{-1} \sum_{C(E)} \mathbf{D}_{C(E)}^{V} \mathbf{P}_{C(E)}^{\prime}
$$

Substituting these pressure-velocity correction relations into difference continuity equation (29a) will lead to the pressure correction equation:

$$
\begin{gathered}
\sum_{E(C)} \sum_{C(E)}\left(\rho A_{E(C) x} \mathbf{C}_{E(C)}^{-1} \mathbf{D}_{C(E)}^{V}+\rho A_{E(C) y} \mathbf{C}_{E(C)}^{-1} \mathbf{D}_{C(E)}^{U}\right) \mathbf{P}_{C(E)}^{\prime} \\
=-\sum_{E(C)}\left(\rho A_{x} \widetilde{\mathbf{U}}+\rho A_{y} \widetilde{\mathbf{V}}\right)_{E(C)}
\end{gathered}
$$




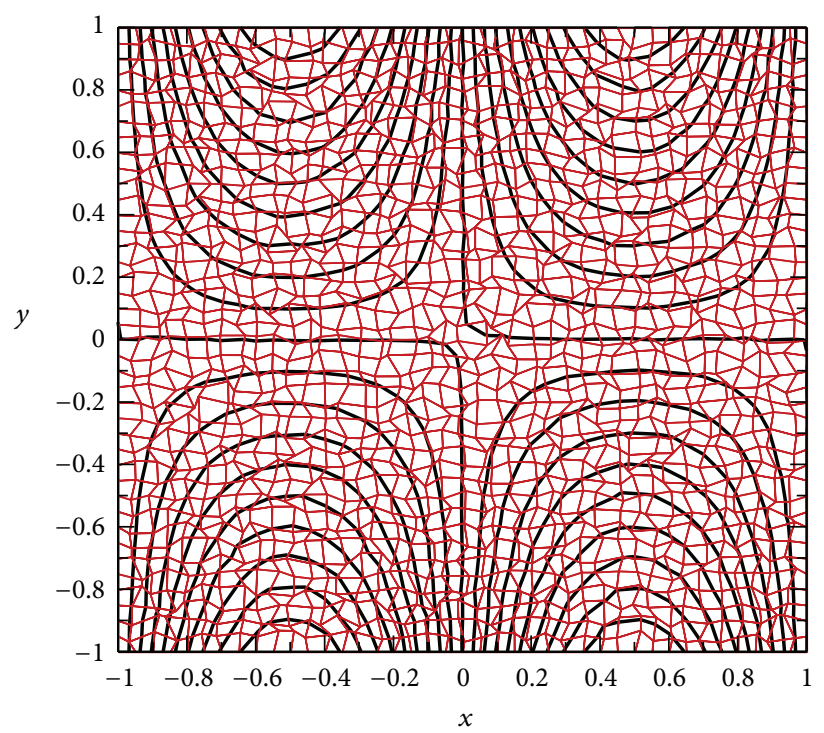

(a) Mesh D

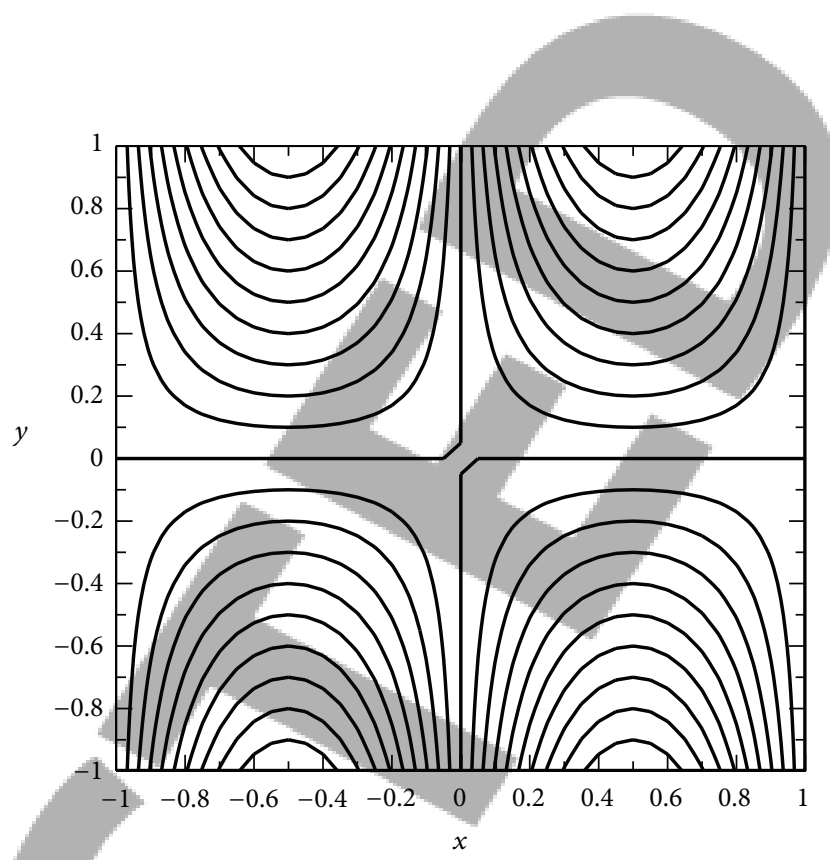

(b) Exact

FIGURE 13: Solution contours in two-dimensional second test problem.

With the given difference momentum and pressure correction equations, simulation can be carried out with the standard SIMPLE procedure.

\section{Numerical Verification}

5.1. One-Dimensional Problems. The first test problem to be solved is the linear convectiondiffusion without source term with boundary conditions

$$
\Phi(0)=0, \quad \Phi(1)=1 .
$$

Figure 5 illustrates the maximum computational error with $\rho u \ell / \mu=500$ and characteristic length $\ell=1$. As is shown in this figure, the computational results confirm the numerical analyses. Implementation of a constant scheme factor of $1 \leq \omega<3$ can yield a second-order accurate solution. Solution with $\omega=3$ becomes third-order accurate. In the range of large cell Reynolds number, calculations with $\omega=$ 2 and $\omega=3$ depict larger errors due to the occurrence of oscillatory solution. However, these errors are still less than those for the conventional CD and QUICK schemes. Therefore, we can summarize that the present formulation can provide much more accurate numerical solution than the conventional schemes.

The second test problem considered in this category is a steady Burger's equation:

$$
\frac{d}{d x}\left(\frac{1}{2} \Phi^{2}-\frac{d \Phi}{d x}\right)=0 .
$$

The corresponding exact solution reads

$$
\Phi(x)=-\phi_{c} \tanh \left(\frac{\phi_{c} x}{2}\right) .
$$

The calculated profiles of solution variable and its spatial gradient along with the exact solution are shown in Figure 6. The calculations are preformed with 40 computational cells. In this figure, the normalizing value, $\phi_{o}$, is defined as the solution at the left boundary, $x=-1$, or $\phi_{o}=\Phi(-1)$. Formulation with $1 \leq \omega \leq 3$ can provide almost identically well-predicted solution profiles as well as its spatial gradient. The computational errors of solution variable with respect to characteristic cell Reynolds number, $R_{c}=\phi_{c} h$, are demonstrated in Figure 6(b) for $\phi_{c}=500$. It clearly shows that the proposed scheme can achieve second-order accurate simulation.

The last test problem is a modeled equation to simulate water transport in membrane [16], which reads

$$
\frac{d}{d x}\left(\Phi-\Phi \frac{d \Phi}{d x}\right)=0
$$

In this model equation, the nonlinear effect is caused by the diffusion coefficient. The interested domain is defined in the range of $\ln 2 \leq x \leq 1$ and the boundary conditions are assigned as

$$
\Phi(\ln 2)=0, \quad \Phi(1)=1 .
$$

The exact solution for this equation can be obtained as

$$
x=\Phi-\ln (1+\Phi)+\ln 2 .
$$

Difficulty in simulation arises due to the solution singular behavior at the inlet boundary, $x=\ell n 2$, where the value of solution variable vanishes and its spatial gradient becomes infinite. Figure 7 demonstrates the computational profiles of solution variable and its spatial gradient. These calculations are performed with 20 computational cells. It is shown that all the scheme factors can yield very accurate results. 


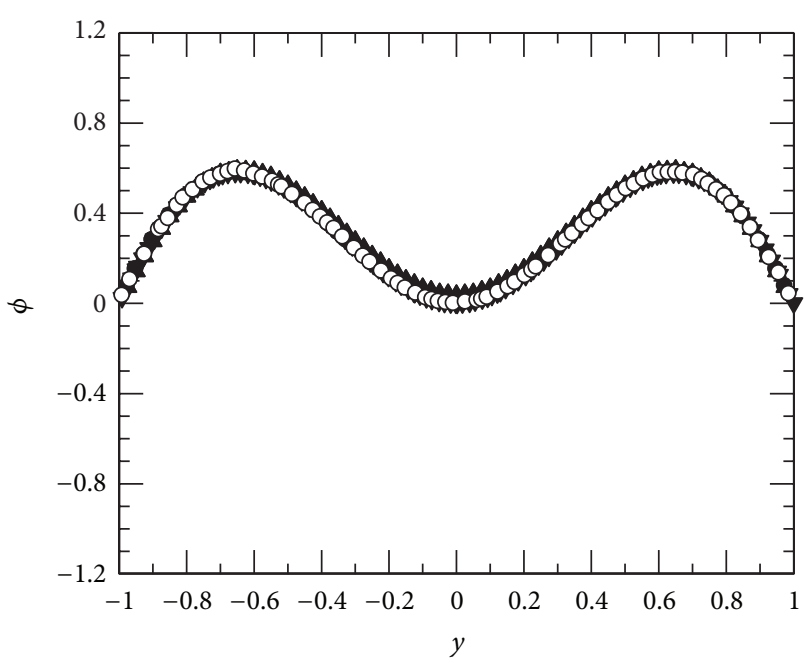

(a)

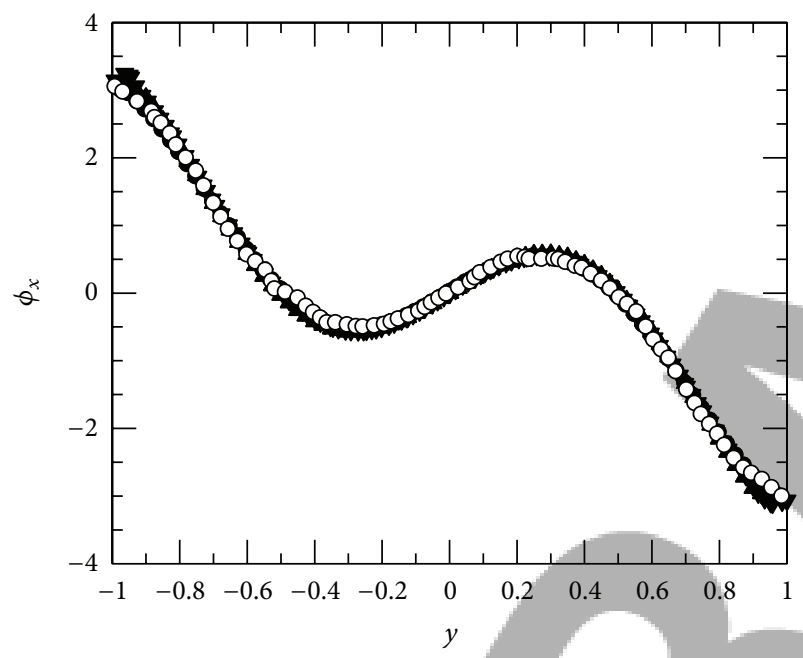

(b)

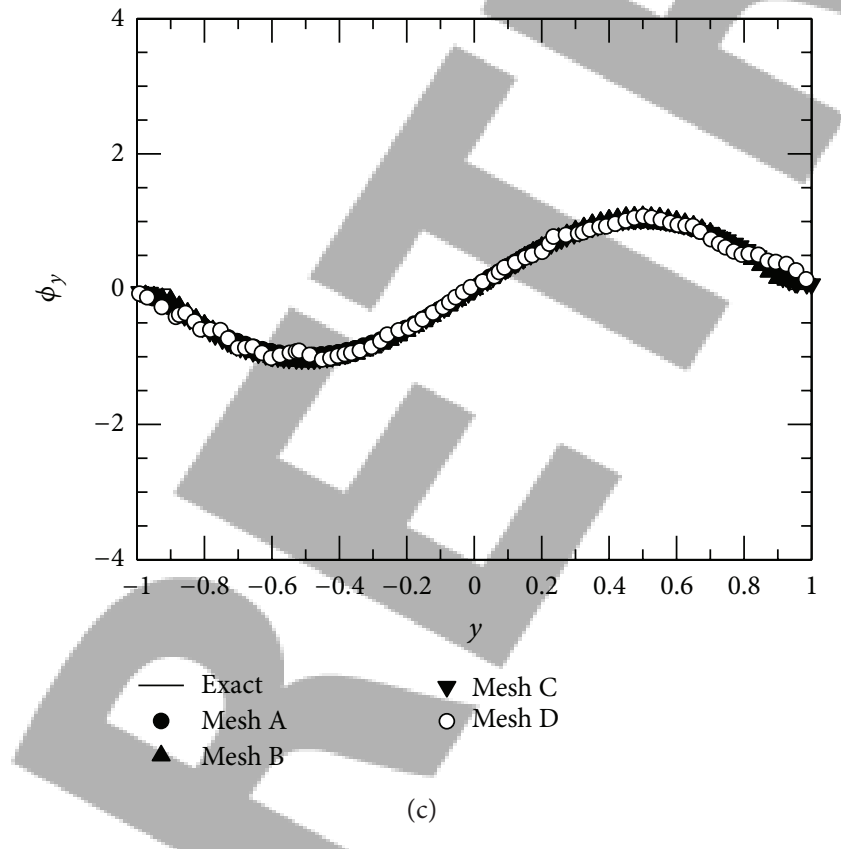

FIGURE 14: Solution profile along $x=y$ in the two-dimensional third test problem.

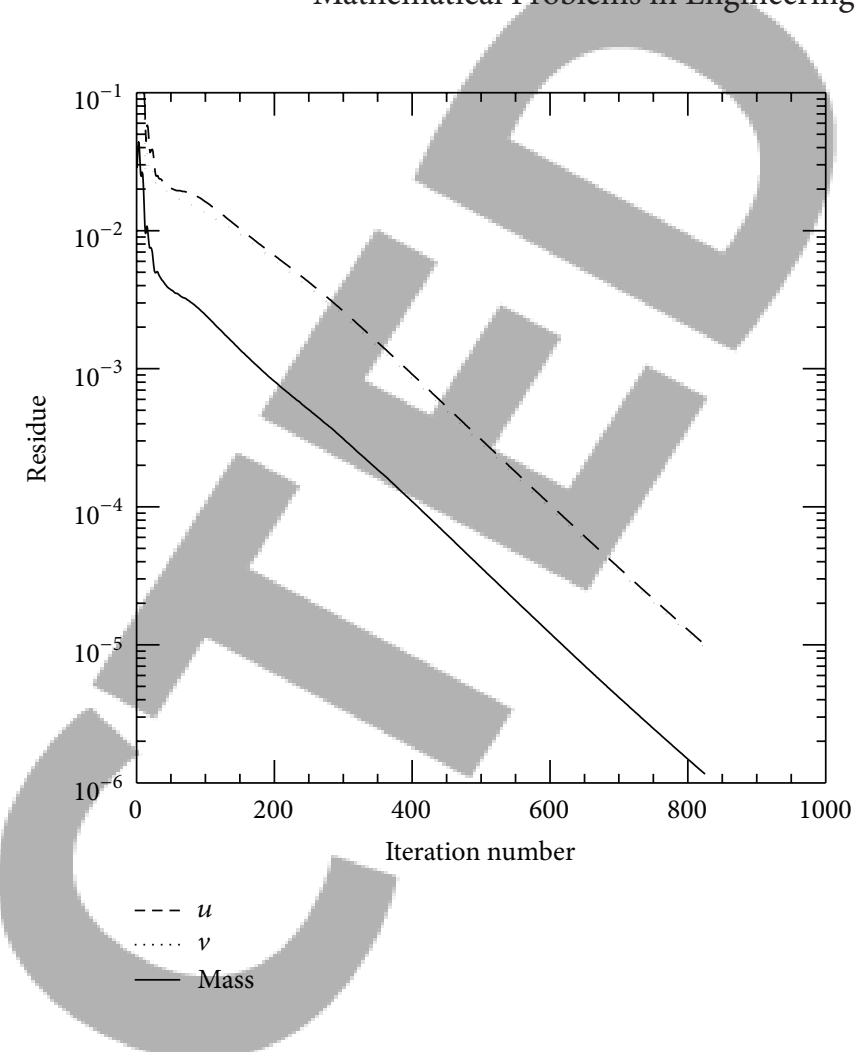

FIGURE 15: Convergence history of a typical lip-driven cavity flow simulation.

5.2. Two-Dimensional Scalar Problems. To investigate the effects of grid distribution, especially in unstructured grid arrangements, we adopted four types of grid arrangement for comparison. These are rectangular (mesh A), regularly triangular grid (mesh B), unstructured triangular grid (mesh C), and randomly quadrilateral grid (mesh D). The regularly triangular grid is derived by dividing a uniform rectangular cell into two triangles and the unstructured triangular grid is generated by the advancing front method [17]. The randomly distributed quadrilateral grid is also derived from a rectangular grid by randomly varying its grid locations:

$$
\begin{aligned}
& x=x_{\mathrm{rec}}+\alpha\left(\psi_{x}-0.5\right) \Delta x, \\
& y=y_{\mathrm{rec}}+\alpha\left(\psi_{y}-0.5\right) \Delta y,
\end{aligned}
$$

where $x_{\text {rec }}$ and $y_{\text {rec }}$ are the original grid location coordinates in rectangular grid, $\psi_{x}$ and $\psi_{y}$ are the random numbers in the range of $0<\psi_{x}, \psi_{y}<1$, and $\alpha$ is a grid distortion parameter. Figure 8 (a) shows a typical $40 \times 40$ rectangular grid, while Figure $8(\mathrm{~b})$ a regularly triangular grid derived from this rectangular mesh. Figure 8(c) illustrates an unstructured triangular grid system. This grid system is generated based on uniformly distributed grid density same as in Figures 8(a) and 8 (b). The resulting randomly quadrilateral grid with $\alpha=0.8$ is displayed in Figure 8(d). 


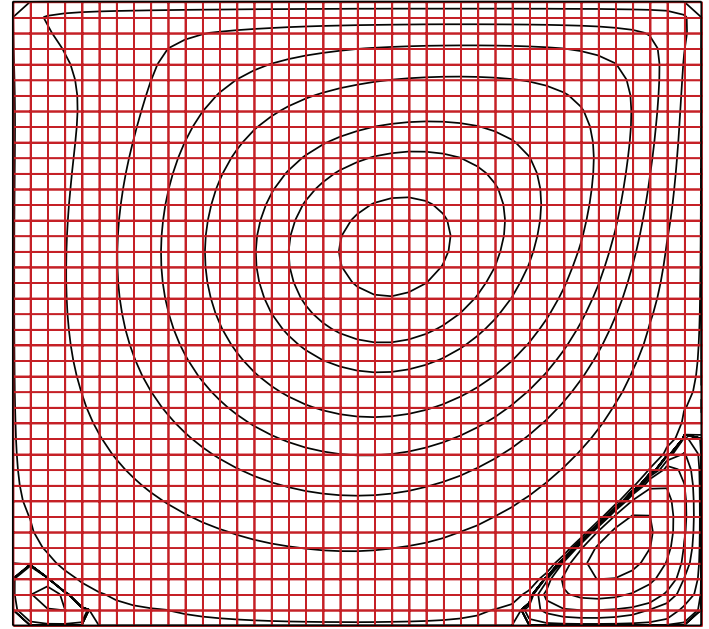

(a) Mesh R

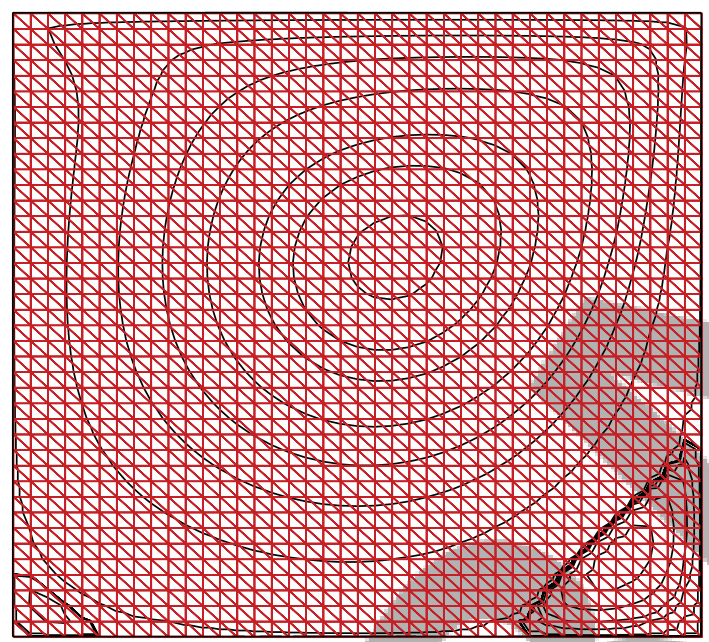

(c) Mesh T

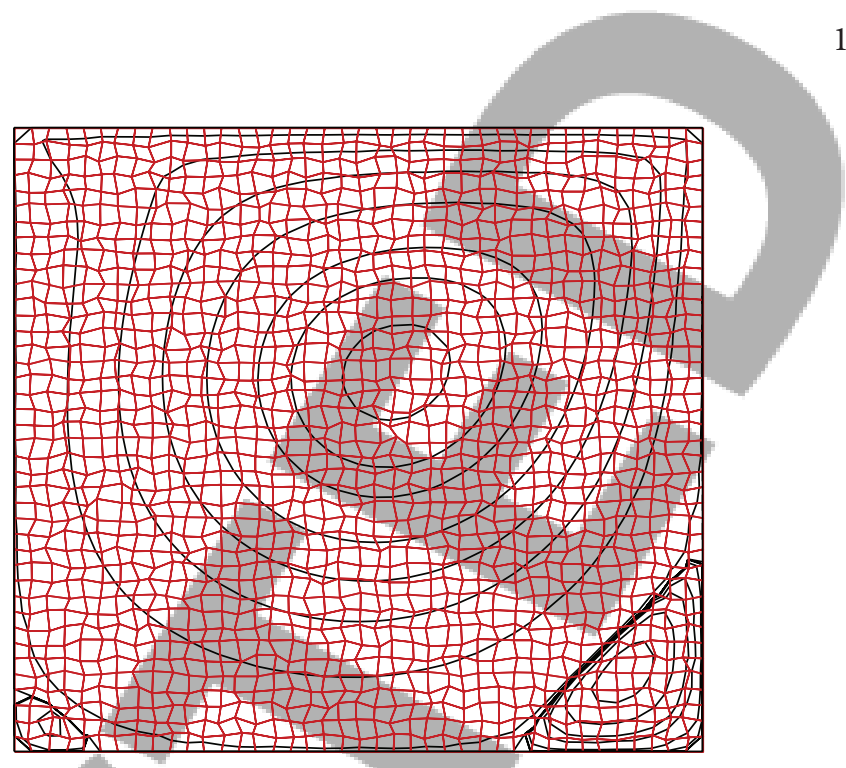

(b) Mesh $\mathrm{R}^{\prime}$

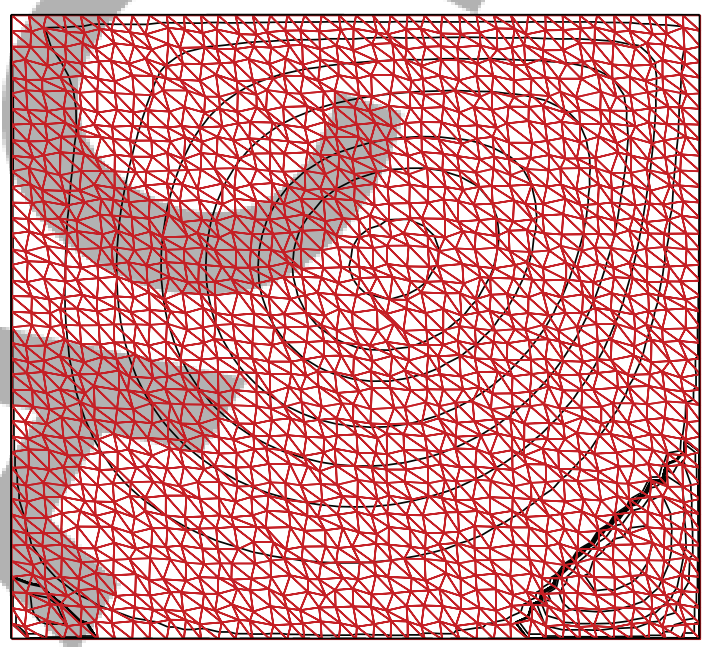

(d) Mesh $\mathrm{T}^{\prime}$

FIGURE 16: Grid arrangement and streamlines in lip-driven cavity flow computations.

The first test problem is a nonlinear convection-diffusion problem:

$$
\Phi \cos \theta \frac{\partial \Phi}{\partial x}+\Phi \sin \theta \frac{\partial \Phi}{\partial y}-\mu\left(\frac{\partial^{2} \Phi}{\partial x^{2}}+\frac{\partial^{2} \Phi}{\partial y^{2}}\right)=0
$$

with the exact solution

$$
\Phi(x, y)=-\tanh \left(\frac{x \cos \theta+y \sin \theta}{2 \mu}\right) .
$$

Fluid viscosity of $\mu=0.02$ is employed. Figure 9(a) depicts the solution contours imbedded with the adopted grid arrangement (mesh D), which can be compared with the exact solution in Figure 9(b). As evident in these figures, the computational results are in good agreement with the exact solution. This observation can be reinforced by the comparisons of solution profiles along $y=0$, which are shown in Figures $10(\mathrm{a})$ and 10(b). In these figures, both the solution variable and its spatial gradients obtained with various grid arrangements are compared with the exact solution. It is clearly shown that well-predicted results can be obtained regardless of grid arrangement.

The second problem is a linear convection-diffusion problem:

$$
\cos \theta \frac{\partial \Phi}{\partial x}+\sin \theta \frac{\partial \Phi}{\partial y}-\mu\left(\frac{\partial^{2} \Phi}{\partial x^{2}}+\frac{\partial^{2} \Phi}{\partial y^{2}}\right)=0
$$

which may simulate cross-stream diffusion effects. If the streamwise diffusion is neglected, we can obtain the approximate exact solution

$$
\Phi(x, y)=\operatorname{erf}\left[\frac{1}{2 \sqrt{\mu}} \frac{-x \sin \theta+y \cos \theta}{\sqrt{(x+1) \cos \theta+(y+1) \sin \theta+\varepsilon_{d}}}\right],
$$

where erf[] is the error function. In our computations, Fluid viscosity of $\mu=0.01$ is employed. Meanwhile, $\varepsilon_{d}=$ 


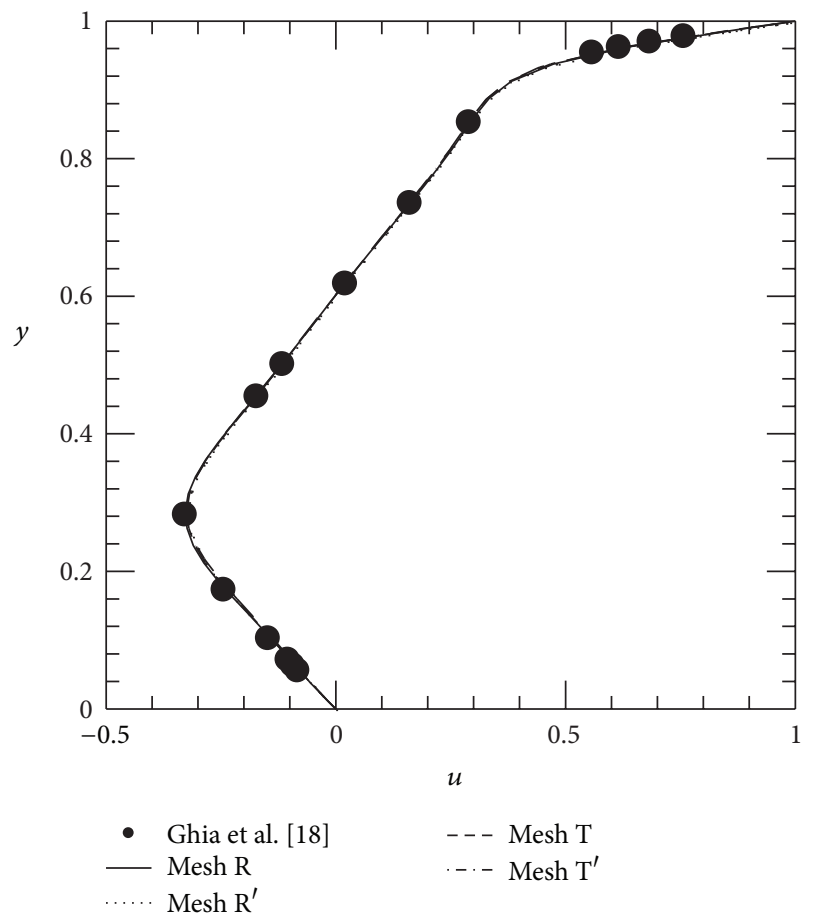

(a) $x$-component

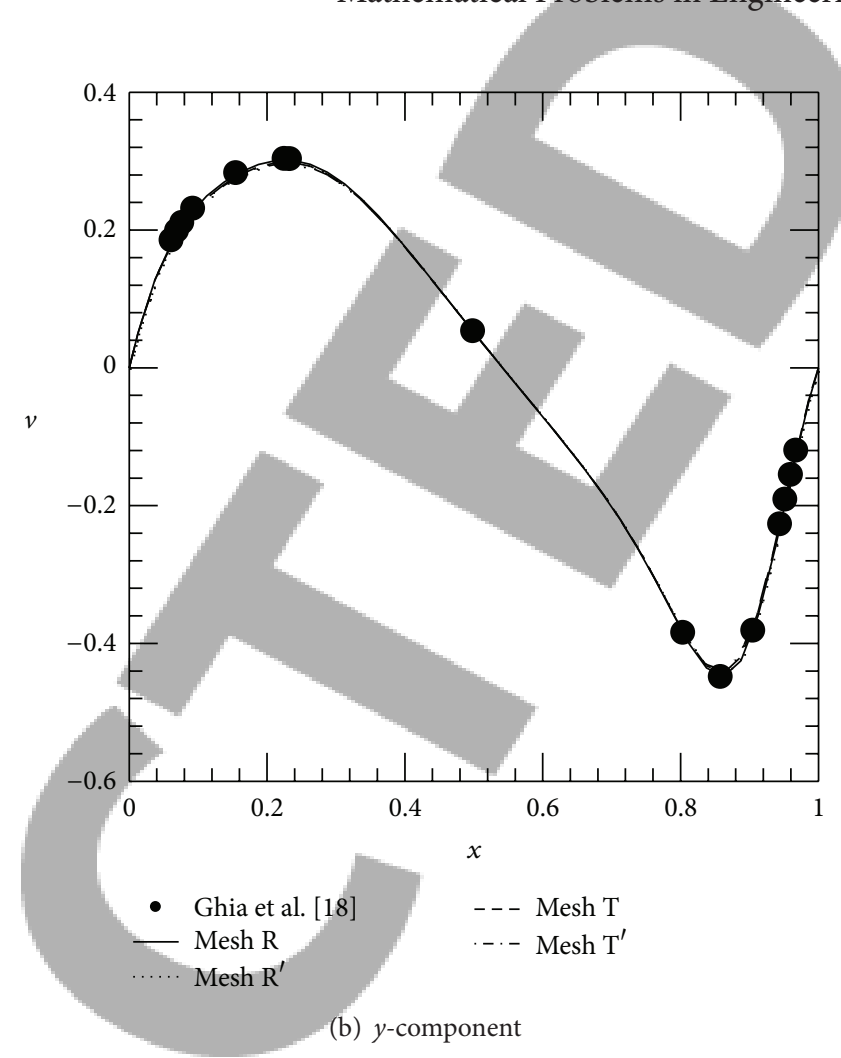

(b) $y$-component

FIGURE 17: Velocity profile across cavity geometrical center.

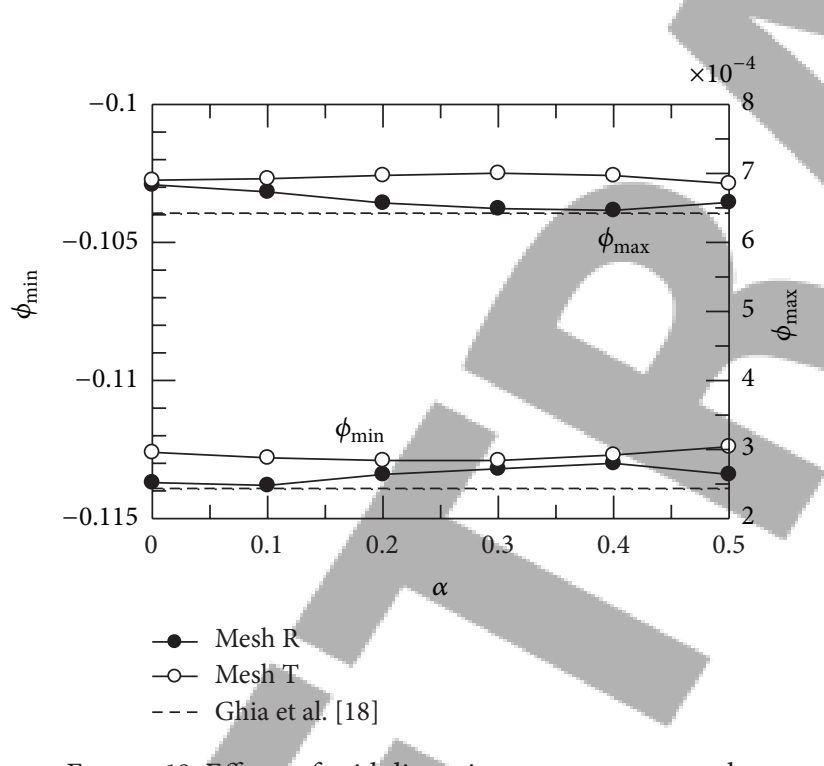

FIGURE 18: Effects of grid distortion on vortex strength.

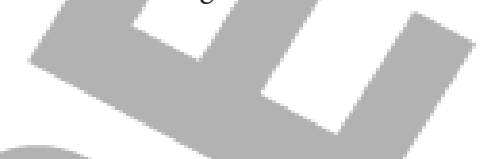

$10^{-3}$ is assigned to avoid the possible solution singularity. Figure 11(a) demonstrates the computational solution contours accompanied by adopted grid arrangement (mesh D) and Figure 11(b) depicts the approximate exact solution for $\theta=45^{\circ}$. Detailed solution profiles along $x=0$ for the computational and approximate exact solutions are displayed in Figures 12(a) and 12(b). As shown in these figures, very agreed results can be obtained for all grid arrangements.

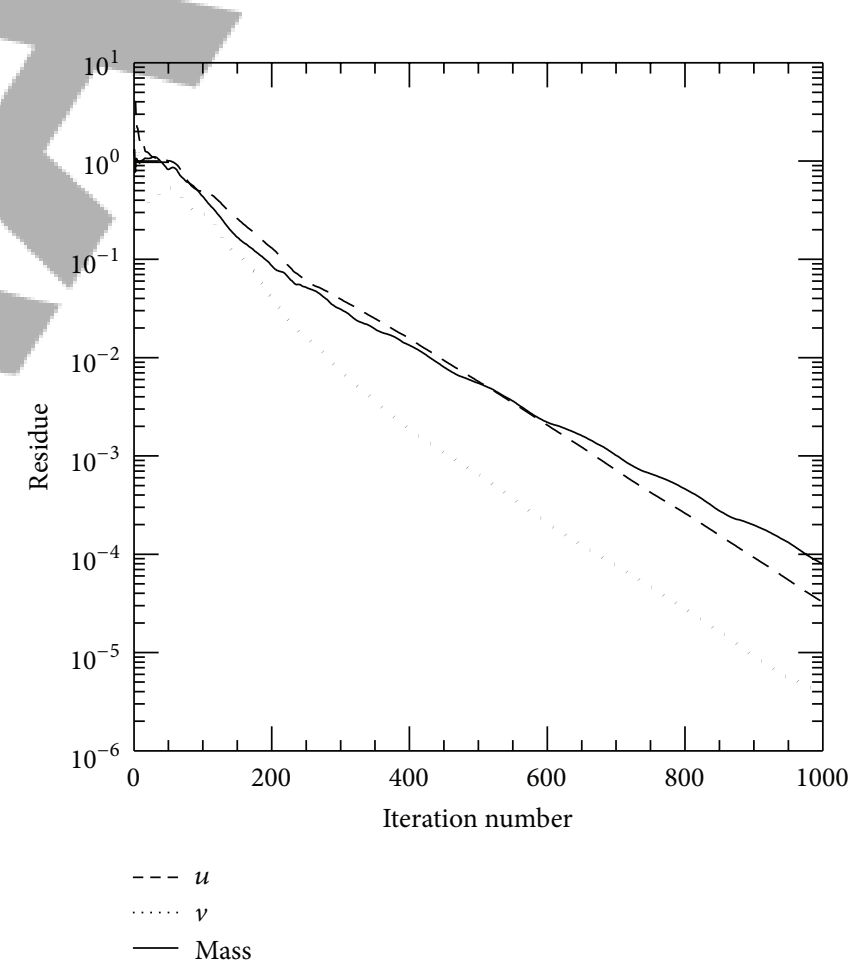

FIGURE 19: Convergence history of a typical backward facing step flow simulation.

The last test problem considered in this study is a convection-diffusion problem in a rotating flow field: 


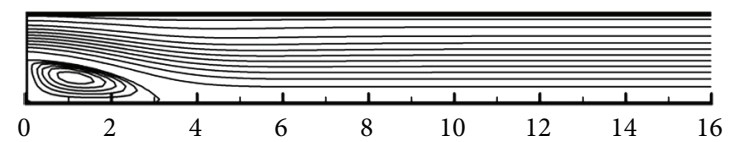

(a) $\mathrm{Re}_{\text {step }}=100$

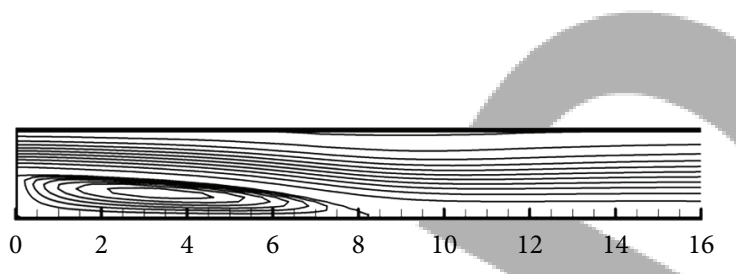

(b) $\mathrm{Re}_{\text {step }}=389$

FIGURE 20: Streamlines in backward facing step flow.

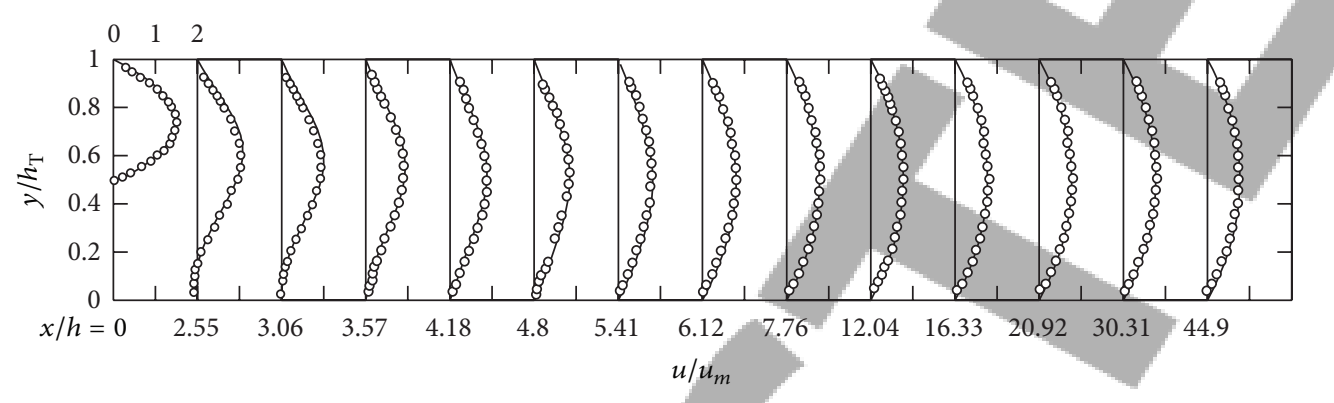

(a) $\operatorname{Re}_{\text {step }}=100$

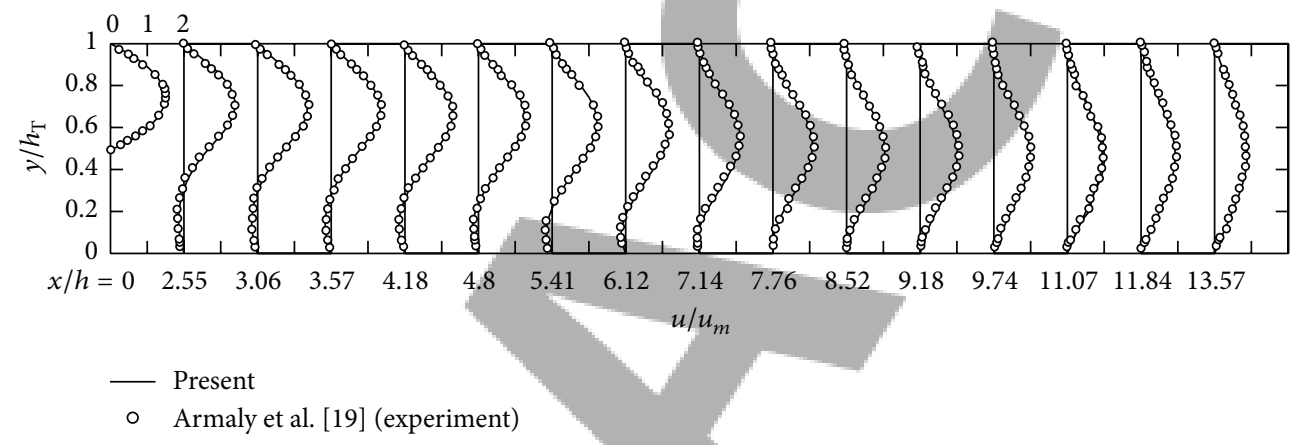

(b) $\mathrm{Re}_{\text {step }}=389$

FIGURE 21: Comparison of axial velocity profiles.

with the velocity field

$$
u \frac{\partial \Phi}{\partial x}+v \frac{\partial \Phi}{\partial y}-\mu\left(\frac{\partial^{2} \Phi}{\partial x^{2}}+\frac{\partial^{2} \Phi}{\partial y^{2}}\right)=S
$$

Source term in the governing equation (39a) is assigned to yield the exact solution

$$
\Phi(x, y)=y \sin (\pi x)
$$

Computations are conducted with dimensionless fluid viscosity of $\mu=0.01$. Figure 13(a) shows the solution contours with the adopted grid system (mesh D), while Figure 13(b) depicts the exact solution contours for comparison. Comparisons of the computational solution profiles along the line of $x-y=0$ on various types of grid arrangements and exact solution are shown in Figure 14. From these figures, it is clearly shown that both the solution variable and its gradients can yield reasonable results as compared with the exact solution.
5.3. Lid-Driven Cavity Flow. The flow Reynolds number based on driving velocity, fluid viscosity, and cavity width is $\operatorname{Re}_{\mathrm{ld}}=400$ in present computations. To investigate the effects of grid distribution, four types of grid arrangement are employed. They are rectangular (mesh $\mathrm{R}$ ), randomly quadrilateral grid (mesh $\mathrm{R}^{\prime}$ ), regularly triangular grid (mesh $\mathrm{T}$ ), and randomly triangular grid (mesh $\mathrm{T}^{\prime}$ ). Figure 15 illustrates the convergence history for a typical computation with $40 \times 40$ uniform rectangular grid (mesh $\mathrm{R}$ ). Equation residuals of the mass, $x$-momentum $(u)$ and $y$-momentum $(v)$ equations are depicted in this figure. From the monotonely decreasing equation residues depicted in this figure, it is clearly shown that the present formulation can provide a very stable computation. The random meshes $\left(\mathrm{R}^{\prime}\right.$ and $\left.\mathrm{T}^{\prime}\right)$ are obtained with the grid distortion parameter $\alpha=0.5$. With this mesh density, Figures 16(a), 16(b), 16(c), and 16(d) demonstrate the resulting streamlines as well as the adopted grid arrangements for mesh $\mathrm{R}, \mathrm{R}^{\prime}, \mathrm{T}$, and $\mathrm{T}^{\prime}$, respectively. From these figures, quite agreed streamlines can be observed irrespective of grid arrangement. This observation can be reinforced by the velocity profile distributions across the cavity geometrical center in Figures 17(a) and 17(b). All computational results 


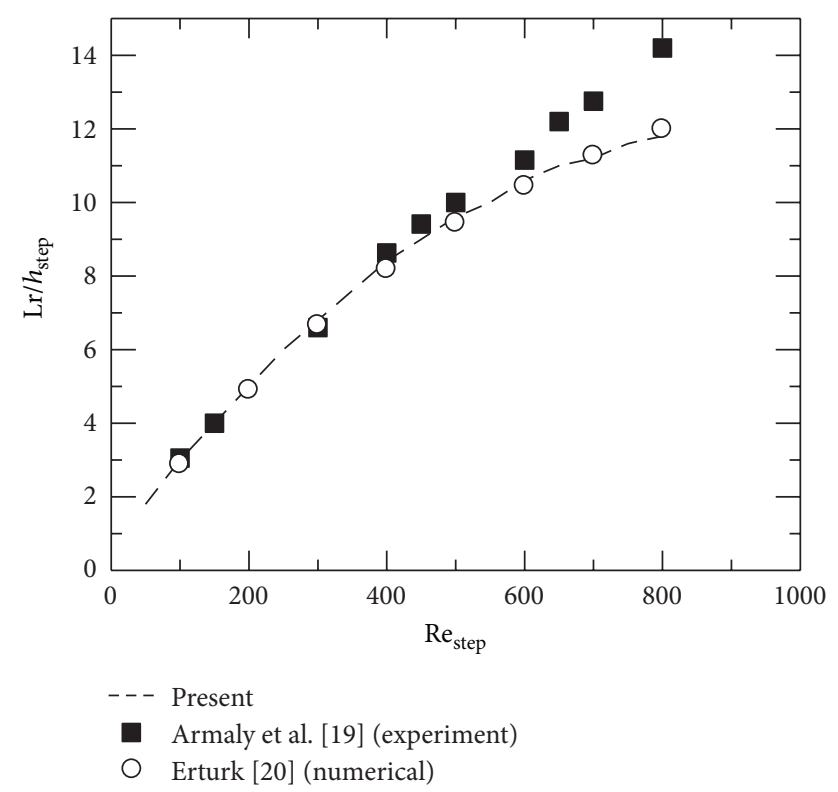

FiguRE 22: Comparison of reattachment length.

are in very good agreement with the benchmark solution [18]. Figure 18 illustrates the vortex strength in terms of adopted grid distortion. Only slight variations in vortex strength are observed for $0 \leq \alpha \leq 0.5$.

5.4. Backward Facing Step Flow. The second test problem considered in the present study is a backward facing step flow with expansion ratio, $\left(h_{\text {in }}+h_{\text {step }}\right) / h_{\text {in }}=1.942$, where $h_{\text {in }}$ and $h_{\text {step }}$ are the inlet and step heights, respectively. In our computations, the grid distribution in the $x$-direction is defined by the following rule:

$$
\begin{array}{cl}
0 \leq x \leq 15 h_{\text {step }} & \text { uniform } \Delta x, \\
15 h_{\text {step }}<x \leq 30 h_{\text {step }} & \text { expansion } \Delta x, \\
30 h_{\text {step }}<x \leq 60 h_{\text {step }} & \text { uniform } \Delta x .
\end{array}
$$

The initial grid spacing is set as $\Delta x=0.2 h_{\text {step }}$ and expansion ratio is 1.1. Uniform grid is set in the $y$-direction with $\Delta y=0.1 h_{\text {step }}$. Figure 19 shows the convergence history of a typical flow simulation, which demonstrates a very stable computation. The resulting streamlines of computations with Reynolds numbers of $\mathrm{Re}_{\text {step }}=100$ and 389 are displayed in Figures 20(a) and 20(b), respectively. This Reynolds number is defined by the inlet mean velocity, fluid viscosity, and inlet hydraulic diameter. Comparisons with available experimental data [19] on axial velocity are shown in Figures 21(a) and $21(\mathrm{~b})$ for $\mathrm{Re}_{\text {step }}=100$ and 389, respectively. In these figures, the axial velocity has been normalized by the mean velocity in the duct, $u_{m}$. Very good agreements are observed between computational and experimental results. Figure 22 illustrates the resulting reattachment length in our simulation along with experimental and other numerical results for comparison. The numerical results provided by Erturk [20] were conducted with a very fine grid, $\Delta y=0.01\left(h_{\text {in }}+h_{\text {step }}\right)$. As shown in this figure, quite agreed results can be observed with the present formulation.

\section{Conclusion}

In the present study, we have successfully developed a feasible solution procedure to simulate incompressible fluid flow in complicated domains. It is put into effect by discretizing the incompressible Navier-Stokes equations with primitive variables on staggered polygonal grids. The discretization procedure of its kernel scheme has been unveiled for convectiondiffusion equation. Its main feature is to directly calculate variable gradients with their own governing equation without further interpolation from solution values at neighboring computational nodes. This process will yield a highresolution discretization with compact stencil in difference equations. Meanwhile, the adoption of staggered grid will eliminate the occurrence of checkerboard pressure field in the resulting solution for its own sake. Pressure-velocity coupling problem in incompressible flow calculation is resolved by the SIMPLE algorithm. Finally, the proposed solution procedure is employed to solve several classic benchmark problems. From the computational results, it can be concluded that the present formulation will be one of the feasible tools to simulate incompressible flow problems.

\section{Nomenclature}

\section{A: Area vector}

a: Coefficient in difference equation

$b$ : Coefficient in difference equation

$c$ : Coefficient in difference equation

$d$ : Coefficient in difference equation

$\mathrm{CV}$ : Control volume

e: Unit vector

f: Numerical flux function vector

$\widehat{\mathrm{f}}$ : Flux function vector

$h$ : $\quad$ Grid size in $x$-direction

$h_{T}$ : Total step height

$\ell$ : Characteristic length

$n_{v}$ : Number of faces in a computational cell

$p$ : Solution gradient in $x$-direction

$q$ : Solution gradient in $y$-direction

Re: Reynolds number

$\mathrm{R}_{h}$ : Cell Reynolds number

r: Position vector

$r$ : Scheme increasing factor

$S$ : Source term

$\bar{s}$ : Average source term

$u$ : Velocity component in $x$-direction

$v$ : Velocity component in $y$-direction

$V$ : Volume of a computational cell

v: Velocity vector

$x$ : Horizontal coordinate

$y:$ Vertical coordinate.

\section{Greek Symbols}

$\alpha$ : Grid distortion parameter

$\Delta$ : Difference

$\theta$ : Flow inclined angle 
$\xi$ : Local coordinate variable

$\eta$ : Local coordinate variable

$\mu$ : Fluid viscosity

$\rho$ : Fluid density

$\Phi$ : Dependent variable

$\phi$ : Numerical solution of $\Phi$

$\psi$ : Random number

$\omega$ : Scheme factor.

\section{Subscripts}

c: Continuity

cr: Critical

ex: Exact

ld: Lid-driven cavity flow

$m$ : Control surface index

p: Computational cell

step: Backward facing step flow

$u$ : $\quad x$-direction momentum

$v: \quad y$-direction momentum

$x: \quad x$-direction

$y: \quad y$-direction.

\section{References}

[1] Y. H. Hwang, "Solution of the steady Navier-Stokes equations by the pressure correction method in the ALE grid," Numerical Heat Transfer B, vol. 23, no. 2, pp. 237-255, 1993.

[2] Y. H. Hwang, "Arbitrary domain velocity analyses for the incompressible Navier-Stokes equations," Journal of Computational Physics, vol. 110, no. 1, pp. 134-149, 1994.

[3] Y. H. Hwang, "Calculations of incompressible flow on a staggered triangular grid, part I: mathematical formulation," Numerical Heat Transfer B, vol. 27, no. 3, pp. 323-336, 1995.

[4] Y. H. Hwang, "Calculations of incompressible flow on a staggered triangular grid, part II: applications," Numerical Heat Transfer B, vol. 27, no. 3, pp. 337-353, 1995.

[5] Y. H. Hwang, "Stability and accuracy analyses for the incompressible Navier-Stokes equations on the staggered triangular grid," Numerical Heat Transfer B, vol. 32, no. 3, pp. 321-349, 1997.

[6] T. J. Barth and P. O. Frederickson, "Higher-order solution of the Euler equations on unstructured grids using quadratic reconstruction,” AIAA Paper 90-0013, 1990.

[7] C. Ollivier-Gooch and M. Van Altena, "A high-order-accurate unstructured mesh finite-volume scheme for the advectiondiffusion equation," Journal of Computational Physics, vol. 181, no. 2, pp. 729-752, 2002.

[8] F. Moukalled and M. Darwish, "New bounded skew central difference scheme, part I: formulation and testing," Numerical Heat Transfer B, vol. 31, no. 1, pp. 91-110, 1997.

[9] Y. H. Hwang, "Development of a compact and accurate discretization for incompressible navier-stokes equations based on an equation-solving solution gradient, part I: kernel scheme fundamentals," Numerical Heat Transfer B, vol. 58, no. 3, pp. 145-169, 2010.

[10] Y. H. Hwang, "Development of a compact and accurate discretization for incompressible navier-stokes equations based on an equation-solving solution gradient, part II: formulation on unstructured polygonal grids," Numerical Heat Transfer B, vol. 58, no. 3, pp. 170-192, 2010.

[11] Y. H. Hwang, "Development of a compact and accurate discretization for incompressible Navier-Stokes equations based on an equation-solving solution gradient, part III: fluid flow simulations on staggered polygonal grids," Numerical Heat Transfer B, vol. 58, no. 3, pp. 193-215, 2010.

[12] Y. H. Hwang, "Development of a compact and accurate discretization for incompressible Navier-Stokes equations based on an equation-solving solution gradient: gradient limiters for high-reynolds-number flows," Numerical Heat Transfer B, vol. 58, no. 6, pp. 440-460, 2010.

[13] Y. H. Hwang, "An equation-solving solution gradient strategy for high-resolution and compact discretization of hyperbolic conservation laws," Numerical Heat Transfer B, vol. 59, no. 5, pp. 381-408, 2011.

[14] W. Shyy, "A study of finite difference approximations to steadystate, convection-dominated flow problems," Journal of Computational Physics, vol. 57, no. 3, pp. 415-438, 1985.

[15] S. V. Patankar, Numerical Heat Transfer and Fluid Flow, Hemisphere, Washington, DC, USA, 1980.

[16] A. A. Kulikovsky, "Simple and accurate scheme for nonlinear convection-diffusion equation," Journal of Computational Physics, vol. 173, no. 2, pp. 716-729, 2001.

[17] S. H. Lo, "A new mesh generation scheme for arbitrary planar domains," International Journal for Numerical Methods in Engineering, vol. 21, no. 8, pp. 1403-1426, 1985.

[18] U. Ghia, K. N. Ghia, and C. T. Shin, "High-Re solutions for incompressible flow using the Navier-Stokes equations and a multigrid method," Journal of Computational Physics, vol. 48, no. 3, pp. 387-411, 1982.

[19] B. F. Armaly, F. Durst, J. C. F. Pereira, and B. Schoenung, "Experimental and theoretical investigation of backward-facing step flow," Journal of Fluid Mechanics, vol. 127, pp. 473-496, 1983.

[20] E. Erturk, "Numerical solutions of 2-D steady incompressible flow over a backward-facing step, part I: High Reynolds number solutions," Computers and Fluids, vol. 37, no. 6, pp. 633-655, 2008 . 